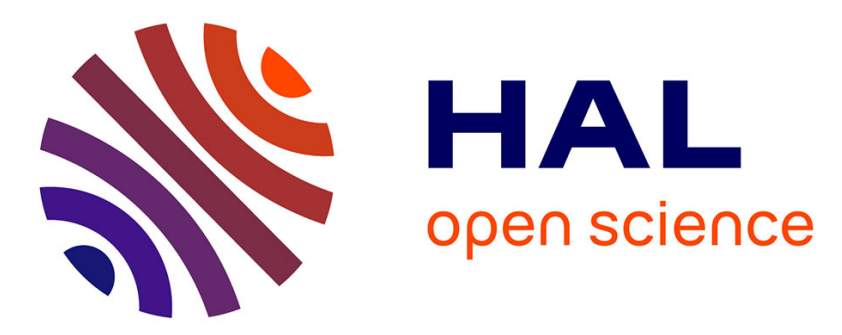

\title{
Tensor-valued random fields for meso-scale stochastic model of anisotropic elastic microstructure and probabilistic analysis of representative volume element size
}

Christian Soize

\section{To cite this version:}

Christian Soize. Tensor-valued random fields for meso-scale stochastic model of anisotropic elastic microstructure and probabilistic analysis of representative volume element size. Probabilistic Engineering Mechanics, 2008, 23 (2-3), pp.307-323. 10.1016/j.probengmech.2007.12.019 . hal-00685154

\section{HAL Id: hal-00685154 \\ https://hal.science/hal-00685154}

Submitted on 4 Apr 2012

HAL is a multi-disciplinary open access archive for the deposit and dissemination of scientific research documents, whether they are published or not. The documents may come from teaching and research institutions in France or abroad, or from public or private research centers.
L'archive ouverte pluridisciplinaire HAL, est destinée au dépôt et à la diffusion de documents scientifiques de niveau recherche, publiés ou non, émanant des établissements d'enseignement et de recherche français ou étrangers, des laboratoires publics ou privés. 


\title{
Tensor-valued random fields for meso-scale stochastic model of anisotropic elastic microstructure and probabilistic analysis of representative volume element size
}

\author{
C. Soize \\ Université Paris-Est, Laboratoire de Mecanique \\ 5 Bd Descartes, 77454 Marne-la-Vallee, France \\ E-mail address: christian.soize@univ-Paris-Est.fr \\ Tel.: 3316095 7661, Fax: 33160957799
}

\begin{abstract}
The main objective of this paper is to present a generic meso-scale probability model for a large class of random anisotropic elastic microstructures in order to perform a parametric analysis of the Representative Volume Element (RVE) size. This new approach can be useful for a direct experimental identification of random anisotropic elastic microstructures when the standard method cannot easily be applied to anisotropic elastic microstructures. Such a RVE is used to construct the macroscopic properties in the context of stochastic homogenization. The probability analysis is not performed as usual for a given particular random microstructure defined in terms of its constituents. Instead, it is performed for a large class of random anisotropic elastic microstructures. For this class, the probability distribution of the random effective stiffness tensor is explicitly constructed. This allows a full probability analysis of the RVE size to be carried out and its convergence to be studied. The procedure of homogenization is based on a homogeneous Dirichlet condition on the boundary of the RVE. The probability model used for the stiffness tensor-valued random field of the random anisotropic elastic microstructure is an extension of the model recently introduced by the author for elliptic stochastic partial differential operators. The stochastic boundary value problem is numerically solved by using the stochastic finite element method. The probability analysis of the RVE size is performed by studying the probability distribution of the random operator norm of the random effective stiffness tensor with respect to the spatial correlation length of the random microstructure.
\end{abstract}

Keywords: Microstructures, probability and statistics, anisotopic material, elastic material, inhomogeneous material

\section{Introduction}

In linear elasticity, the objective of stochastic homogenization is to construct the macroscopic elastic properties of random elastic microstructures. Such a stochastic homogenization is an approximation based (1) on the calculation of the solution of the boundary value problem relative to the Representative Volume Element (RVE) with a periodic, Dirichlet or Neumann boundary condition and (2) on calculating the spatial averaging of this solution on the RVE. Stochastic homogenization can be applied if the scale of the random microstructure is sufficiently small with respect to macroscopic dimensions. This means that RVE size has to be small with respect to macroscopic dimensions and sufficiently large with respect to the scale of fluctuations in the microstructure. Such a definition is not really sufficient and must be mathematically defined in the context of a probability model of the microstructure.

The homogenization of random heterogeneous materials with random microstructures and the calculation of the macroscopic properties have received considerable attention in the past three decades; see for instance (Kröner, 1971), (Nemat-Nasser and Hori, 1999), (Milton, 2002) and (Torquato, 2002). The first mathematical results concerning stochastic homogenization are attributed to (Sanchez-Palencia, 1980) and (Papanicolaou and Varadhan, 1981). Many papers and books have been published in the field of stochastic homogenization, on calculating effective properties and their bounds, constructing non-local effective constitutive equations, such as (Kröner, 1977),(Sab, 1992), (Jikov et al, 1994), (Andrews and Wright, 1998), (Nemat-Nasser and Hori, 1999). 
Often, the probabilistic model of a random microstructure (such as a composite constituted of several constituents) can directly be constructed from the geometry and mechanical properties of its constituents. This is the case for the class of random heterogeneous materials whose microstructures can be modelled as a distribution of inclusions or cavities of well-defined geometry in a given matrix. This is also the case of random heterogeneous materials having a cellular struture for which the probabilistic model is directly constructed using cell statistics, random field models, percolation and clustering, and for which the n-point probability distribution functions can be deduced from the knowledge of the different phases constituting the microstructure; see for instance (Torquato and Stell, 1985), (Andraud et al., 1997), (Torquato, 1997), (Nemat-Nasser and Hori, 1999), (Quintanilla, 1999), (Ostoja-Starzewski, 1998), (Kachanov et al., 2001), (Milton, 2002), (Torquato, 2002). Generally, the statistics-based bounding techniques only use the lower-order statistics (first- and second-order moments) and the probability distributions which give the detailed probabilistic information are not taken into account.

The random microstructure can be homogenized if there is a RVE size which is "small" with respect to macroscopic dimensions, i.e. if the random fluctuations of the random effective stiffness tensor around the statistical mean value of the random effective stiffness tensor is "negligible". Representative volume element size has received a particular attention; see for instance (Nemat-Nasser and Hori, 1999), (Ren and Zheng, 2004). These works are developed for elastic composite materials with firmly-bonded phases. In (Drugan and Willis, 1996), the RVE size is related to the "convergence of the mean value of the effective tensor" and in (Sab and Nedjar, 2005), the RVE size has to be such that the random fluctuations are "small enough".

The objectives of this paper are detailed below.

(A) The construction of the system of marginal probability distributions for any composite using the geometry and the mechanical properties of its constituents requires the knowledge of all the $n$-point probability functions which can be not so easy to deduce from theoretical considerations and/or from experimental measurements in particular for anisotropic elastic microstructure. For some random anisotropic elastic microstructures, it can be difficult (1) to deduce the probabilistic model of the microstructure from the probabilistic model of its constituents and (2) to identify and/or to validate the probabilistic model from experimental measurements performed on several specimens and using mathematical statistics for estimating the probability models of random fields under consideration. This is the reason why it can be interesting to propose an additional approach for identifying the probabilistic model of a random anisotropic elastic microstructure which cannot easily be deduced (and experimentally identified) from its constituents. Such a new approach is proposed in this paper.

(B) The main idea of this paper is then to directly introduce a meso-scale probabilistic model of the random anisotropic elastic microstructure, which is not deduced from the probabilistic models of its constituents. Such a meso-scale probabilistic model must verify fundamental mathematical properties to obtain a physical model of any anisotropic elastic microstructure. The random anisotropic elastic microstructure (for instance a mortar constituted of a cement paste with embedded sand particles, some porous media such as plaster boards, some cortical bones, some biological membranes and more generally, some living tissues, etc) is then modelled at the mesoscale by an equivalent random continuous anisotropic elastic medium which is completely defined by its local stiffness fourth-order tensor-valued random field $\mathbf{x} \mapsto \mathbb{C}(\mathbf{x})=\left\{\mathbb{C}_{i j k h}(\mathbf{x})\right\}_{i j k h}$. The random field $\mathbb{C}$ is then constituted of 21 mutually dependent real-valued random fields modelling the anisotropic microstructure at the meso-scale level. The theory proposed allows strong anisotropic random fluctuations to be taken into account. The great interest of such a direct construction of a meso-scale probabilistic model of the random anisotropic elastic microstructure is 
the capability to identify the parameters of the random field $\mathbf{x} \mapsto \mathbb{C}(\mathbf{x})$. Such an experimental identification can be performed using displacement measurements of tested specimens on the boundary of the meso-scale subdomain of the RVE by using field measurements deduced from digital image processing and solving an inverse stochastic problem. It is then necessary to choose a stochastic representation of $\mathbf{x} \mapsto \mathbb{C}(\mathbf{x})$ in a class of random fields for which only a few parameters are required to define its system of marginal probability distributions. Therefore, the inverse problem related to such an experimental identification of $\mathbf{x} \mapsto \mathbb{C}(\mathbf{x})$ is more feasible. In addition, such a class of random fields $\mathbf{x} \mapsto \mathbb{C}(\mathbf{x})$ must be constructed using only the available information and not "hypothetical" information for which no statistics are available or for which the number of experimental specimens is too small to obtain a good convergence of the statistical estimators. For the tensor-valued random field $\mathbf{x} \mapsto \mathbb{C}(\mathbf{x})$, the largest class can be constructed using as available information: the symmetry properties, the mean value $\mathbf{x} \mapsto \mathbb{C}(\mathbf{x})$ which is assumed to be known and a stochastic non-uniform ellipticity condition for the corresponding linear elasticity stochastic differential operator. Clearly, any random anisotropic elastic microstructure belongs to this class.

As explained above, the prime objective of this paper is not to analyse a particular random isotropic or anisotropic microstructure described in terms of its constituents, but is to propose (when the standard method cannot easily be applied to anisotropic elastic microstructures) a new way which could be useful for a direct experimental identification of random anisotropic elastic microstructures introducing a meso-scale probabilistic model. It should be noted that the comparison of the proposed approach with the standard approach is not so easy to perform. The previously published works are mainly devoted to define the RVE size for which a deterministic effective stiffness tensor can be constructed and used, and are generally not attached to construct the probability distribution of the random effective stiffness tensor for random anisotropic elastic microstructures. In addition, many previous works deal with isotropic or orthotropic microstructures and cannot be compared with the present anisotropic case developed in this paper.

(C) The second objective of this paper is to perform a parametric probabilistic study of the RVE size with respect to the spatial correlation lengths of the proposed meso-scale probabilistic model. The parametric study of the RVE size is performed in function of the intensity of the stochastic fluctuations of $\mathbf{x} \mapsto \mathbb{C}(\mathbf{x})$ and in function of its correlation lengths. Such a study is also useful to get information on the RVE size for which stochastic fluctuations are still significant and consequently, can be measured. Then the results presented in this paper could allow the meso-scale probabilistic model to be identified from meso- or macro-scale measurements solving an inverse stochastic problem.

(C.1) The probability analysis of the RVE size which is proposed for constructing the effective (or macroscopic) stiffness of random anisotropic elastic microstructures is performed as follows. Let $Z=\left\|\mathbf{A}^{\text {eff }}\right\| / m_{\left\|\mathbf{A}^{\text {eff }}\right\|}$ be the real-valued random variable in which the random variable $\left\|\mathbf{A}^{\text {eff }}\right\|$ is the operator norm of the random effective stiffness tensor $\mathbf{A}^{\text {eff }}$ and where $m_{\left\|\mathbf{A}^{\text {eff }}\right\|}=$ $E\left\{\left\|\mathbf{A}^{\text {eff }}\right\|\right\}$ is the mean value with $E$ the mathematical expectation. The probability distribution (the cumulative distribution function) of the random variable $Z$ is explicitly constructed and allows a full probability analysis of the RVE size to be performed. The use of the Chebychev inequality for the random variable $Z$ also allows the convergence of the random effective stiffness tensor to be studied with the coefficient of variation of $Z$. Nevertheless, if such an approach allows convergence in probability to be analysed, it only gives an upper bound of the probability when convergence is not completely reached (it should be noted that such a difficulty is circumvented by using the full probability analysis proposed in this paper). 
(C.2) Another objective of this work is to quantify the RVE size in terms of probability level. This means that the probability distribution of the random fluctuations of the random effective stiffness tensor is effectively calculated. For such an analysis, there are two equivalent approaches: (1) the three spatial correlation lengths of the tensor-valued random field $\mathbf{x} \mapsto \mathbb{C}(\mathbf{x})$ are fixed, and then the size of the RVE is taken as a variable parameter, or (2) the three spatial correlation lengths are taken as variable parameters and then the size of the RVE is fixed. The present analysis uses the second approach and the RVE has dimensions $1 \times 1 \times 1$.

(D) The strategy of the developments presented in this work is the following:

(D.1) Since the proposed meso-scale probability model of the random anisotropic microstructure is general and since we are interested in constructing the probability distribution of the random effective stiffness tensor, the random solution of the stochastic boundary value problem cannot be exactly constructed with an analytical method. It is therefore constructed using the stochastic finite element method and an adapted stochastic solver.

(D.2) The approximation of the random effective stiffness tensor can be constructed by averaging the random operator on the RVE using one of the three following boundary conditions: periodic, Dirichlet or Neumann boundary conditions. For a periodic microstructure, the Hill-Mandel condition which indicates that both the Dirichlet and Neumann problems are needed to establish the RVE size can be used (see for instance (Nemat-Nasser and Hori, 1999)). Such a criterion allows the RVE size to be defined in calculating the effective stiffness tensor for both the Dirichlet and Neumann boundary conditions. The RVE size is then such that the two calculated effective stiffness tensors must be "equal" for a given precision. However, for a random microstructure, this criterion can be replaced by another criterion related to the random fluctuations of the random effective stiffness tensor calculated either with the Dirichlet condition or with the Neumann condition. The random fluctuations must then be "small" for a given precision either for the Dirichlet problem or for the Neumann problem. This property has mathematically been analysed: a recent work published by (Bourgeat and Piatnitski, 2004) shows that the three approximations constructed with the three boundary conditions converge to the same effective stiffness tensor for a sufficiently general probabilistic model. Since the limit is the same in the stochastic case, the result is indifferent to the type of the chosen boundary conditions. Finally, since the mechanical analysis at the macro-scale is often performed in terms of the displacement field, one then has chosen the homogeneous Dirichlet condition on the boundary of the RVE in order to limit the development of the present work.

(D.3) The RVE size is analysed in the context of the standard theory of local homogenization for which the random effective stiffness tensor is constant in space (local theory). This is an approximation of non-local theories (see for instance: (Kröner, 1971), (Beran and McCoy, 1970), (Nemat-Nasser and Hori, 1999)). Nevertheless, the development of a full stochastic non-local theory for the 3D-anisotropic cases which would be based on the use of the proposed meso-scale probabilistic model for random anisotropic elastic microstructures is not the purpose of this paper.

(E) The paper is organized as follows:

(E.1) Section 2 deals with the standard theory concerning the macroscopic properties of a random anisotropic elastic microstructure. The usual procedure of homogenization with a given homogeneous Dirichlet condition on the boundary of the RVE is recalled (see for instance (NematNasser and Hori, 1999), (Zaoui, 2002)) and the probabilistic model of the random anisotropic microstructure is presented. This section is introduced to define the notation and the random quantities for the stochastic case. 
(E.2) In Sections 3 and 4, the largest class of all the tensor-valued random fields $\mathbf{x} \mapsto \mathbb{C}(\mathbf{x})$ corresponding to the meso-scale probability model of random anisotropic elastic microstructures is presented. This largest class is an extension of the subclass presented in (Soize, 2006). The detailed construction of this largest class of random fields cannot be duplicated here and only the final result and the main mathematical properties are summarized in order to simplify the reading. Note that the extension of the mathematical proof concerning the stochastic non-uniform ellipticity condition for this largest class is straightforward.

(E.3) Section 5 is devoted to the weak formulation of the stochastic boundary value problem (BVP), to the use of the stochastic finite elements method to construct a finite approximation of this stochastic BVP and finally, to the stochastic solver. Then, the probabilistic quantities for the random effective stiffness tensor can be calculated. It should be noted that the usual proof of the existence of a second-order random solution, based on the use of a uniform ellipticity condition and a uniform boundness condition for the fourth-order tensor-valued random field $\mathbf{x} \mapsto \mathbb{C}(\mathbf{x})$, cannot be used. This is due to the introduction of a more general non uniform ellipticity condition which is a more realistic probabilistic hypothesis. Consequently, the existence of a second-order solution is studied.

(E.4) Finally, Section 6 deals with the probabilistic analysis of the RVE size which is performed by numerical simulation and is limited to the subclass of the meso-scale probabilistic models for random anisotropic elastic microstructures.

\section{Macroscopic properties of a random anisotropic microstructure}

Consider a random microstructure constituted of a random heterogeneous anisotropic elastic linear medium. The random local (or microscopic) constitutive equation is written as

$$
\boldsymbol{\sigma}(\mathbf{x})=\mathbb{C}(\mathbf{x}): \boldsymbol{\varepsilon}(\mathbf{x})
$$

which means $\boldsymbol{\sigma}_{j k}(\mathbf{x})=\mathbb{C}_{j k \ell m}(\mathbf{x}) \boldsymbol{\varepsilon}_{\ell m}(\mathbf{x})$ where $\mathbf{x}=\left(x_{1}, x_{2}, x_{3}\right)$ is a point of the Representative Volume Element (RVE) which is a $3 \mathrm{D}$ bounded open domain $\Omega$ in $\mathbb{R}^{3}$ and where $\mathbf{x} \mapsto \mathbb{C}(\mathbf{x})$ is the fourth-order tensor-valued random field allowing the elastic properties of the random microstructure to be characterized. Let $\mathbf{x} \mapsto \mathbf{D}(\mathbf{x})=\left(D_{1}(\mathbf{x}), D_{2}(\mathbf{x}), D_{3}(\mathbf{x})\right)$ be the random local displacement field. In Eq. (1), $\mathbf{x} \mapsto \boldsymbol{\varepsilon}(\mathbf{x})=\varepsilon(\mathbf{D}(\mathbf{x}))$ is the random local strain tensor field such that $\varepsilon_{\ell m}(\mathbf{D}(\mathbf{x}))=\frac{1}{2}\left(\partial D_{\ell}(\mathbf{x}) / \partial x_{m}+\partial D_{m}(\mathbf{x}) / \partial x_{\ell}\right)$, and $\mathbf{x} \mapsto \boldsymbol{\sigma}_{j k}(\mathbf{x})$ is the random local stress tensor field. The random effective (or macroscopic) stress and strain tensors are usually defined as the average in the RVE of the random local stress and strain tensor fields,

$$
<\boldsymbol{\sigma}>=\frac{1}{|\Omega|} \int_{\Omega} \boldsymbol{\sigma}(\mathbf{x}) d \mathbf{x}, \quad<\boldsymbol{\varepsilon}>=\frac{1}{|\Omega|} \int_{\Omega} \boldsymbol{\varepsilon}(\mathbf{x}) d \mathbf{x} .
$$

It should be noted that $\langle\boldsymbol{\sigma}\rangle$ and $\langle\boldsymbol{\varepsilon}>$ are random tensors.

\subsection{Localization}

As explained in Section 1-(D.2), the homogeneous Dirichlet condition on the boundary of the RVE is used. The localization is then done with a given random effective strain $\underline{\varepsilon}$ on the boundary $\partial \Omega$ of the RVE which is independent of $\mathbf{x}$. One then has $\mathbf{D}(\mathbf{x})=\underline{\boldsymbol{\varepsilon}} \mathbf{x}$ on $\partial \Omega$ (i.e. $\left.D_{\ell}(\mathbf{x})=\underline{\boldsymbol{\varepsilon}}_{\ell m} x_{m}\right)$ in which the given tensor $\underline{\boldsymbol{\varepsilon}}$ is independent of $\mathbf{x}$ and such that $\underline{\boldsymbol{\varepsilon}}=<\boldsymbol{\varepsilon}>$. Consequently, for a given random effective strain $\underline{\varepsilon}$ on $\partial \Omega$, the random local displacement field 
D in the microstructure $\Omega$ can be constructed by solving the following stochastic boundary value problem (BVP) in $\Omega$,

$$
\begin{aligned}
& -\operatorname{div} \boldsymbol{\sigma}=0 \text { in } \Omega, \\
& \mathbf{D}(\mathbf{x})=\underline{\boldsymbol{\varepsilon}} \mathbf{x} \text { on } \partial \Omega,
\end{aligned}
$$

in which $\{\operatorname{div} \boldsymbol{\sigma}(\mathbf{x})\}_{j}=\partial \boldsymbol{\sigma}_{j k}(\mathbf{x}) / \partial x_{k}$ and where the random local constitutive equation is defined by Eq. (1). Since the solution $\mathbf{D}$ of Eqs. (3) and (4) depends linearly on $\underline{\varepsilon}$, the random local strain tensor can be written as

$$
\varepsilon(\mathbf{D}(\mathbf{x}))=H(\mathbf{x}): \underline{\boldsymbol{\varepsilon}},
$$

in which the fourth-order tensor-valued random field $\mathbf{x} \mapsto H(\mathbf{x})$ corresponds to the strain localization associated with the stochastic BVP defined by Eqs. (3) and (4). Since $\varepsilon(\mathbf{D}(\mathbf{x}))$ and $\underline{\varepsilon}$ are symmetric tensors, from Eq. (5), it can be deduced that

$$
\uplus_{j k \ell m}(\mathbf{x})=\uplus_{k j \ell m}(\mathbf{x})=\uplus_{j k m \ell}(\mathbf{x})
$$

In order to construct the random field $H$, for all $\ell$ and $m$ in $\{1,2,3\}$, the second-order tensors $g^{\ell m}$ are introduced such that

$$
g_{j k}^{\ell m}=\frac{1}{2}\left(\delta_{j \ell} \delta_{k m}+\delta_{j m} \delta_{k \ell}\right)
$$

in which $\delta_{j \ell}$ is the Kronecker symbol. From Eq. (7), it can be verified that $g_{j k}^{\ell m}=g_{j k}^{m \ell}=g_{k j}^{\ell m}$ and that

$$
\underline{\boldsymbol{\varepsilon}}=\underline{\boldsymbol{\varepsilon}}_{l m} g^{\ell m}
$$

For all $\ell$ and $m$ in $\{1,2,3\}$, let $\mathbf{D}^{\ell m}$ be the random local displacement field which is the solution of the following stochastic BVP in $\Omega$,

$$
\begin{aligned}
-\operatorname{div} \boldsymbol{\sigma}^{\ell m}=0 & \text { in } \Omega, \\
\mathbf{D}^{\ell m}(\mathbf{x})=g^{\ell m} \mathbf{x} & \text { on } \partial \Omega,
\end{aligned}
$$

in which (see Eq. (1)), $\boldsymbol{\sigma}^{\ell m}(\mathbf{x})=\mathbb{C}(\mathbf{x}): \varepsilon\left(\mathbf{D}^{\ell m}(\mathbf{x})\right)$. From Eqs. (3)-(4) and (5), it can be deduced that the solution $\mathbf{D}^{\ell m}$ of Eqs. (9)-(10) is such that $\varepsilon\left(\mathbf{D}^{\ell m}(\mathbf{x})\right)=H(\mathbf{x}): g^{\ell m}$ which, using Eqs. (7) and (8), yields

$$
\uplus_{j k \ell m}(\mathbf{x})=\varepsilon_{j k}\left(\mathbf{D}^{\ell m}(\mathbf{x})\right)
$$

From Eqs. (11) and (2), it can be deduced that $<\uplus_{j k \ell m}>=<\varepsilon_{j k}\left(\mathbf{D}^{\ell m}(\mathbf{x})\right)>$. Comparing Eqs. (4) and (10) and using Eq. (2) yield $<\varepsilon_{j k}\left(\mathbf{D}^{\ell m}(\mathbf{x})\right)>=g_{j k}^{\ell m}$. It can then be deduced that

$$
<\mathbb{H}_{j k \ell m}>=g_{j k}^{\ell m}
$$




\subsection{Random effective stiffness tensor}

The fourth-order random effective stiffness tensor $\mathbb{C}^{\text {eff }}$ is defined by the following equation,

$$
<\boldsymbol{\sigma}>=\mathbb{C}^{\mathrm{eff}}:\langle\boldsymbol{\varepsilon}>
$$

which means that $\left\langle\boldsymbol{\sigma}_{j k}>=\mathbb{C}_{j k \ell m}^{\text {eff }}<\boldsymbol{\varepsilon}_{\ell m}>\right.$. Substituting Eq. (5) in Eq. (1) yields $\boldsymbol{\sigma}=\mathbb{C}: \mathbb{H}: \underline{\boldsymbol{\varepsilon}}$, and consequently, $\langle\boldsymbol{\sigma}\rangle=\langle\mathbb{C}: \mathbb{H}\rangle: \underline{\boldsymbol{\varepsilon}}$, which compared to Eq. (13) and taking into account that $\underline{\varepsilon}=<\varepsilon>$ yield

$$
\mathbb{C}^{\mathrm{eff}}=\langle\mathbb{C}: \mathbb{H}\rangle
$$

which means that $\mathbb{C}_{j k \ell m}^{\text {eff }}=<\mathbb{C}_{j k p q} H_{p q \ell m}>$. Due to the symmetry of the fourth-order random local stiffness tensor $\mathbb{C}$, taking into account Eq. (6) and using (Nemat-Nasser and Hori, 1999), the energetic characterization, it can be proven that the fourth-order random effective stiffness tensor $\mathbb{C}^{\text {eff }}$ is symmetric, that is to say,

$$
\mathbb{C}_{j k \ell m}^{\mathrm{eff}}=\mathbb{C}_{k j \ell m}^{\mathrm{eff}}=\mathbb{C}_{j k m \ell}^{\mathrm{eff}}=\mathbb{C}_{\ell m j k}^{\mathrm{eff}}
$$

Consequently, for a given probabilistic model of the fourth-order tensor-valued random field $\mathbf{x} \mapsto \mathbb{C}(\mathbf{x})$ allowing to define the elastic properties of the random microstructure, the fourth-order random effective stiffness tensor $\mathbb{C}^{\text {eff }}$ is calculated by using Eq. (14) in which the fourth-order tensor-valued random field $\mathbf{x} \mapsto \mathbb{H}(\mathbf{x})$ is given by Eq. (11). For all $\ell$ and $m$ in $\{1,2,3\}, \mathbf{D}^{\ell m}$ is the random local displacement field which is the solution of the stochastic BVP defined by Eqs. (9)-(10).

\subsection{Meso-scale probabilistic model for random anisotropic elastic microstructures}

As explained in Section 1-(B), the fourth-order tensor-valued random field $\mathbf{x} \mapsto \mathbb{C}(\mathbf{x})$ corresponding to the meso-scale probabilistic model for the random anisotropic elastic microstructure is introduced. This model will be used to construct the fourth-order random effective stiffness tensor $\mathbb{C}^{\text {eff }}$. For all $\mathbf{x}$ fixed in $\Omega$, the random fourth-order tensor $\mathbb{C}(\mathbf{x})$ has a given mean value, must verify the symmetry property and a stochastic non-uniform ellipticity condition related to positive-definiteness properties. As explained in Section 1, the random field $\mathbb{C}$ is constituted of 21 mutually dependent real-valued random fields and the system of marginal probability distributions of $\mathbb{C}$ is required because the unknown random solution $\mathbf{x} \mapsto \mathbf{D}^{\ell m}(\mathbf{x})$ of the stochastic BVP defined by Eqs. (9)-(10) is a non-linear mapping of the random field $\mathbb{C}$.

The mean value of the random field $\mathbb{C}$ is a deterministic tensor-valued field $\mathbf{x} \mapsto\left\{\underline{\mathbb{C}}_{i j k h}(\mathbf{x})\right\}_{i j k h}$ associated with the mean model of the meso-scale probabilistic model of the random anisotropic elastic microstructure. The probability model has to be such that $E\left\{\mathbb{C}_{i j k h}(\mathbf{x})\right\}=\underline{\mathbb{C}}_{i j k h}(\mathbf{x})$ for all $\mathbf{x}$, where $E$ is the mathematical expectation. The known symmetries, such as monoclinic symmetry, orthotropic symmetry, transverse square symmetry, transversally isotropic symmetry, and isotropic symmetry, can be taken into account with the mean model represented by the tensor $\left\{\underline{\mathbb{C}}_{i j k h}(\mathbf{x})\right\}_{i j k h}$. Nevertheless, this paper deals with the case for which the random fluctuation tensor $\left\{\mathbb{C}_{i j k h}(\mathbf{x})-\underline{\mathbb{C}}_{i j k h}(\mathbf{x})\right\}_{i j k h}$ around the mean tensor is purely anisotropic, without any symmetries.

One presents an extension of the probability model proposed in (Soize, 2004 and 2006) which is based on a non-parametric construction of the random field $\mathbf{x} \mapsto \mathbb{C}(\mathbf{x})$, which only uses the available information. In addition, for all deterministic symmetric second-order real tensor $z$, the uniform elliptic condition $\mathbb{C}_{j k \ell m}(\mathbf{x}) z_{\ell m} z_{j k} \geq c_{0} z_{j k} z_{j k}$ a.s. (almost surely), where 
$c_{0}$ is a deterministic positive constant independent of $\mathbf{x}$, is not introduced to construct this probability model because, in general, such a uniform ellipticity condition does not correspond to available information (objective data). For the proposed probabilistic model, a non uniform ellipticity condition is introduced for the fourth-order tensor-valued random field $\mathbf{x} \mapsto \mathbb{C}(\mathbf{x})$ which corresponds to the available information and which, for all $\ell$ and $m$ fixed in $\{1,2,3\}$, allows the random weak formulation of the stochastic BVP defined by Eqs. (9)-(10) to have a unique second-order random solution $\mathbf{x} \mapsto \mathbf{D}^{\ell m}(\mathbf{x})$.

In order to define the probability model of the tensor-valued random field $\mathbb{C}$, the $(6 \times 6)$ matrix representation $[\mathbf{A}(\mathbf{x})]$ of the fourth-order tensor $\mathbb{C}(\mathbf{x})$ is introduced. Therefore, let $I$ and $J$ be the new indices belonging to $\{1, \ldots, 6\}$ such that $I=(j, k)$ and $J=(\ell, m)$ with the following correspondence: $1=(1,1), 2=(2,2), 3=(3,3), 4=(1,2), 5=(1,3)$ and $6=(2,3)$. Thus, for all $\mathbf{x}$ in $\Omega$, the random $(6 \times 6)$ real matrix $[\mathbf{A}(\mathbf{x})]$ is such that

$$
[\mathbf{A}(\mathbf{x})]_{I J}=\mathbb{C}_{j k \ell m}(\mathbf{x})
$$

For all $\mathbf{x}$ fixed in $\Omega$, due to the symmetry and positive-definiteness properties of the random fourth-order tensor $\mathbb{C}(\mathbf{x})$, it can be deduced that $[\mathbf{A}(\mathbf{x})]$ is a random variable with values in the set $M_{6}^{+}(\mathbb{R})$ of all the $(6 \times 6)$ real symmetric positive-definite matrices. The $M_{6}^{+}(\mathbb{R})$-valued random field $\{[\mathbf{A}(\mathbf{x})], \mathbf{x} \in \Omega\}$, indexed by $\Omega$, defined on the probability space $(\Theta, \mathcal{T}, P)$, is constituted of $6 \times(6+1) / 2=21$ mutually dependent real-valued random fields defining the fourth-order tensor-valued random field $\mathbb{C}$ indexed by $\Omega$.

The mean function $\mathbf{x} \mapsto[\underline{a}(\mathbf{x})]$ of the random field $[\mathbf{A}]$ is assumed to be a given function from $\Omega$ into $M_{6}^{+}(\mathbb{R})$ such that, for all $\mathbf{x}$ fixed in $\Omega$,

$$
E\{[\mathbf{A}(\mathbf{x})]\}=[\underline{a}(\mathbf{x})]
$$

Since $[\underline{a}(\mathbf{x})]$ belongs to $\mathbb{M}_{6}^{+}(\mathbb{R})$, there is an upper triangular invertible matrix $[\underline{L}(\mathbf{x})]$ in $M_{6}(\mathbb{R})$ (the set of all the $(6 \times 6)$ real matrices) such that

$$
[\underline{a}(\mathbf{x})]=[\underline{L}(\mathbf{x})]^{T}[\underline{L}(\mathbf{x})]
$$

It is assumed that $\mathbf{x} \mapsto[\underline{L}(\mathbf{x})]$ is bounded on $\Omega$ and that $\mathbf{x} \mapsto[\underline{a}(\mathbf{x})]$ satisfies the usual uniform ellipticity condition on $\Omega$.

For all $\mathbf{x}$ fixed in $\Omega$, the random matrix $[\mathbf{A}(\mathbf{x})]$ can be written as

$$
[\mathbf{A}(\mathbf{x})]=[\underline{L}(\mathbf{x})]^{T}[\mathbf{G}(\mathbf{x})][\underline{L}(\mathbf{x})]
$$

in which $\mathbf{x} \mapsto[\mathbf{G}(\mathbf{x})]$ is a random field defined on $(\Theta, \mathcal{T}, P)$, indexed by $\mathbb{R}^{3}$, with values in $M_{6}^{+}(\mathbb{R})$, such that for all $\mathbf{x}$ in $\mathbb{R}^{3}$

$$
E\{[\mathbf{G}(\mathbf{x})]\}=[I],
$$

in which $[I]$ is the identity matrix. The random field $[\mathbf{G}]$ is completely defined in Section 3 . 


\section{Probability model of the random fields $[G]$ and $[A]$}

Let $d \geq 1$ and $n \geq 1$ be two given integers. The random field $\mathbf{x}=\left(x_{1}, \ldots, x_{d}\right) \mapsto[\mathbf{G}(\mathbf{x})]$ is indexed by $\mathbb{R}^{d}$ with values in $\mathbb{M}_{n}^{+}(\mathbb{R})$. In Eq. (18), one has $d=3$ and $n=6$. As explained in Section 1-(E.2), the extended probability model presented below is based on the construction and the mathematical analysis of the random field $[\mathbf{G}]$ performed in (soize, 2006). The results which allow the numerical calculation to be performed are summarized below. The random field $\mathbf{x} \mapsto[\mathbf{G}(\mathbf{x})]$ is constructed as a homogeneous and normalized non-Gaussian positive-definite matrix-valued random field, defined on probability space $(\Theta, \mathcal{T}, P)$, indexed by $\mathbb{R}^{d}$, with values in $\mathbb{M}_{n}^{+}(\mathbb{R})$. This random field is constructed as a non-linear mapping of $n(n+1) / 2$ independent second-order centered homogeneous Gaussian random fields $\mathbf{x} \mapsto U_{j j^{\prime}}(\mathbf{x}), 1 \leq j \leq j^{\prime} \leq n$, defined on the probability space $(\Theta, \mathcal{T}, P)$, indexed by $\mathbb{R}^{d}$, with values in $\mathbb{R}$, and named the stochastic germs of the non-Gaussian random field $[\mathbf{G}]$.

\subsection{Random fields $U_{j j^{\prime}}$ as the stochastic germs of the random field $[\mathbf{G}]$}

The stochastic germs are constituted of $n(n+1) / 2$ independent second-order centered homogeneous Gaussian random fields $\mathbf{x} \mapsto U_{j j^{\prime}}(\mathbf{x}), 1 \leq j \leq j^{\prime} \leq n$, defined on the probability space $(\Theta, \mathcal{T}, P)$, indexed by $\mathbb{R}^{d}$, with values in $\mathbb{R}$ and such that

$$
E\left\{U_{j j^{\prime}}(\mathbf{x})\right\}=0 \quad, \quad E\left\{U_{j j^{\prime}}(\mathbf{x})^{2}\right\}=1 .
$$

Consequently, all these random fields are completely and uniquely defined by the $n(n+1) / 2$ autocorrelation functions $R_{U_{j j^{\prime}}}(\boldsymbol{\eta})=E\left\{U_{j j^{\prime}}(\mathbf{x}+\boldsymbol{\eta}) U_{j j^{\prime}}(\mathbf{x})\right\}$ defined for all $\boldsymbol{\eta}=\left(\eta_{1}, \ldots, \eta_{d}\right)$ in $\mathbb{R}^{d}$ and such that $R_{U_{j j^{\prime}}}(0)=1$.

In order to obtain a class having a reasonable number of parameters, these autocorrelation functions are written as $R_{U_{j j^{\prime}}}(\eta)=\rho_{1}^{j j^{\prime}}\left(\eta_{1}\right) \times \ldots \times \rho_{d}^{j j^{\prime}}\left(\eta_{d}\right)$ in which, for all $k=1, \ldots, d$, one has $\rho_{k}^{j j^{\prime}}(0)=1$ and for all $\eta_{k} \neq 0$,

$$
\rho_{k}^{j j^{\prime}}\left(\eta_{k}\right)=4\left(L_{k}^{j j^{\prime}}\right)^{2} /\left(\pi^{2} \eta_{k}^{2}\right) \sin ^{2}\left(\pi \eta_{k} /\left(2 L_{k}^{j j^{\prime}}\right)\right),
$$

in which $L_{1}^{j j^{\prime}}, \ldots, L_{d}^{j j^{\prime}}$ are positive real numbers. Each random field $U_{j j^{\prime}}$ is then mean-square continuous on $\mathbb{R}^{d}$ and it power spectral measure has a compact support. Such a model has $d n(n+1) / 2$ real parameters $L_{1}^{j j^{\prime}}, \ldots, L_{d}^{j j^{\prime}}$ for $1 \leq j \leq j^{\prime} \leq n$ which represent the spatial correlation lengths of the stochastic germs $U_{j j^{\prime}}$.

\subsection{Defining an adapted family of functions}

The construction of the random field $[\mathbf{G}]$ requires the introduction of an adapted family of functions $\{u \mapsto h(\alpha, u)\}_{\alpha>0}$. Let $\alpha$ be a positive real number. The function $u \mapsto h(\alpha, u)$ from $\mathbb{R}$ into $] 0,+\infty\left[\right.$ is introduced such that $\Gamma_{\alpha}=h(\alpha, U)$ is a gamma random variable with parameter $\alpha$ while $U$ is a normalized Gaussian random variable $\left(E\{U\}=0\right.$ and $\left.E\left\{U^{2}\right\}=1\right)$. Consequently, for all $u$ in $\mathbb{R}$, one has

$$
h(\alpha, u)=F_{\Gamma_{\alpha}}^{-1}\left(F_{U}(u)\right) .
$$

in which $u \mapsto F_{U}(u)=\int_{-\infty}^{u} \frac{1}{\sqrt{2 \pi}} e^{-v} d v$ is the cumulative distribution function of the normalized Gaussian random variable $U$. The function $p \mapsto F_{\Gamma_{\alpha}}^{-1}(p)$ from $] 0,1[$ into $] 0,+\infty[$ is the reciprocal function of the cumulative distribution function $\gamma \mapsto F_{\Gamma_{\alpha}}(\gamma)=\int_{0}^{\gamma} \frac{1}{\Gamma(\alpha)} t^{\alpha-1} e^{-t} d t$ of the gamma random variable $\Gamma_{\alpha}$ with parameter $\alpha$ in which $\Gamma(\alpha)$ is the gamma function defined by $\Gamma(\alpha)=\int_{0}^{+\infty} t^{\alpha-1} e^{-t} d t$. 


\subsection{Defining the random field $[\mathbf{G}]$}

The random field $\mathbf{x} \mapsto[\mathbf{G}(\mathbf{x})]$, defined on the probability space $(\Theta, \mathcal{T}, P)$, indexed by $\mathbb{R}^{d}$, with values in $M_{n}^{+}(\mathbb{R})$ is constructed as follows:

(i) Let $\left\{U_{j j^{\prime}}(\mathbf{x}), \mathbf{x} \in \mathbb{R}^{d}\right\}_{1 \leq j \leq j^{\prime} \leq n}$ be the $n(n+1) / 2$ independent random fields introduced in Section 3.1. Consequently, for all $\mathbf{x}$ in $\mathbb{R}^{d}$,

$$
E\left\{U_{j j^{\prime}}(\mathbf{x})\right\}=0 \quad, \quad E\left\{U_{j j^{\prime}}(\mathbf{x})^{2}\right\}=1 \quad 1 \leq j \leq j^{\prime} \leq n .
$$

(ii) Let $\delta$ be the real number, independent of $\mathbf{x}$ and $n$, such that

$$
0<\delta<\sqrt{(n+1)(n+5)^{-1}}<1 .
$$

This parameter which is assumed to be known (resulting, for instance, from an experimental identification solving an inverse problem) allows the dispersion of the random field $[\mathbf{G}]$ to be controlled.

(iii) For all $\mathbf{x}$ in $\mathbb{R}^{d}$, the random matrix $[\mathbf{G}(\mathbf{x})]$ is written as

$$
[\mathbf{G}(\mathbf{x})]=[\mathbf{L}(\mathbf{x})]^{T}[\mathbf{L}(\mathbf{x})]
$$

in which $[\mathbf{L}(\mathbf{x})]$ is the upper $(n \times n)$ real triangular random matrix defined as follows:

(iii.1) For $1 \leq j \leq j^{\prime} \leq n$, the $n(n+1) / 2$ random fields $\mathbf{x} \mapsto[\mathbf{L}(\mathbf{x})]_{j j^{\prime}}$ are independent.

(iii.2) For $j<j^{\prime}$, the real-valued random field $\mathbf{x} \mapsto[\mathbf{L}(\mathbf{x})]_{j j^{\prime}}$, indexed by $\mathbb{R}^{d}$, is defined by $[\mathbf{L}(\mathbf{x})]_{j j^{\prime}}=\sigma U_{j j^{\prime}}(\mathbf{x})$ in which $\sigma$ is such that $\sigma=\delta(n+1)^{-1 / 2}$.

(iii.3) For $j=j^{\prime}$, the positive-valued random field $\mathbf{x} \mapsto[\mathbf{L}(\mathbf{x})]_{j j}$, indexed by $\mathbb{R}^{d}$, is defined by $[\mathbf{L}(\mathbf{x})]_{j j}=\sigma \sqrt{2 h\left(\alpha_{j}, U_{j j}(\mathbf{x})\right)}$ in which $\alpha_{j}=(n+1) /\left(2 \delta^{2}\right)+(1-j) / 2$.

\subsection{A few basic properties of the random field $[\mathbf{G}]$}

The random field $\mathbf{x} \mapsto[\mathbf{G}(\mathbf{x})]$ defined in Section 3.3 is a homogeneous second-order meansquare continuous random field indexed by $\mathbb{R}^{d}$ with values in $M_{n}^{+}(\mathbb{R})$ and its trajectories are almost surely continuous on $\mathbb{R}^{d}$. For all $\mathbf{x} \in \mathbb{R}^{d}$, one has

$$
E\left\{\|\mathbf{G}(\mathbf{x})\|_{F}^{2}\right\}<+\infty \quad, \quad E\{[\mathbf{G}(\mathbf{x})]\}=[I],
$$

in which, for any real matrix $[B],\|B\|_{F}^{2}=\operatorname{tr}\left\{[B]^{T}[B]\right\}$. It can be proven that the newly introduced parameter $\delta$ corresponds to the following definition

$$
\delta=\left\{\frac{1}{n} E\left\{\|[\mathbf{G}(\mathbf{x})]-[I]\|_{F}^{2}\right\}\right\}^{1 / 2},
$$

which shows that

$$
E\left\{\|\mathbf{G}(\mathbf{x})\|_{F}^{2}\right\}=n\left(\delta^{2}+1\right),
$$

in which $\delta$ is independent of $\mathbf{x}$ and $n$. For all $\mathbf{x}$ fixed in $\mathbb{R}^{d}$, the probability density function with respect to the measure $\widetilde{d} G=2^{n(n-1) / 4} \Pi_{1 \leq j \leq k \leq n} d[G]_{j k}$ of the random matrix $[\mathbf{G}(\mathbf{x})]$ is independent of $\mathbf{x}$ and is written as

$$
p_{[\mathbf{G}(\mathbf{x})]}([G])=\mathbb{1}_{\mathbb{M}_{n}^{+}(\mathbb{R})}([G]) \times C_{\mathbf{G}} \times(\operatorname{det}[G])^{(n+1) \frac{\left(1-\delta^{2}\right)}{2 \delta^{2}}} \times \exp \left\{-\frac{(n+1)}{2 \delta^{2}} \operatorname{tr}[G]\right\},
$$


in which $\mathbb{1}_{M_{n}^{+}(\mathbb{R})}([G])$ is equal to 1 if $[G] \in M_{n}^{+}(\mathbb{R})$ and is equal to zero if $[G] \notin \mathbb{M}_{n}^{+}(\mathbb{R})$, where tr is the trace of matrices and where positive constant $C_{\mathbf{G}}$ is such that

$$
C_{\mathbf{G}}=\frac{(2 \pi)^{-n(n-1) / 4}\left(\frac{n+1}{2 \delta^{2}}\right)^{n(n+1)\left(2 \delta^{2}\right)^{-1}}}{\left\{\Pi_{j=1}^{n} \Gamma\left(\frac{n+1}{2 \delta^{2}}+\frac{1-j}{2}\right)\right\}} .
$$

For all $\mathbf{x}$ fixed in $\mathbb{R}^{d}$, Eq. (29) shows that the random variables $\left\{[\mathbf{G}(\mathbf{x})]_{j k}, 1 \leq j \leq k \leq n\right\}$ are mutually dependent. In addition, the system of the marginal probability distributions of the random field $\mathbf{x} \mapsto[\mathbf{G}(\mathbf{x})]$ is completely defined and is not Gaussian.

Let $[\mathbf{B}]$ be a random matrix, defined on $(\Theta, \mathcal{T}, P)$, with values in the set $\mathbb{M}_{m}(\mathbb{R})$ of all the $(m \times m)$ real matrices. For $\theta \in \Theta$, let $[\mathbf{B}(\theta)] \in M_{m}(\mathbb{R})$ be a realization of $[\mathbf{B}]$. The norm $\|\mathbf{B}(\theta)\|$ of $[\mathbf{B}(\theta)]$ induced by the Euclidean norm $\|\mathbf{v}\|$ of $\mathbf{v}$ in $\mathbb{R}^{m}$ is such that

$$
\|\mathbf{B}(\theta)\|=\sup _{\|\mathbf{v}\| \leq 1}\|[\mathbf{B}(\theta)] \mathbf{v}\| \quad, \quad \mathbf{v} \in \mathbb{R}^{m} .
$$

The random variable $\theta \mapsto[\mathbf{B}(\theta)]$ is denoted as $\|\mathbf{B}\|$. There exists a positive constant $c_{0}$ independent of $n$ and independent of $\mathbf{x}$, but depending on $\delta$, such that for all $n \geq 2$ and for all $\mathbf{x} \in \mathbb{R}^{d}$

$$
E\left\{\left\|[\mathbf{G}(\mathbf{x})]^{-1}\right\|^{2}\right\} \leq c_{0}<+\infty .
$$

It should be noted that since $[\mathbf{G}(\mathbf{x})]$ almost surely belongs to $M_{n}^{+}(\mathbb{R})$, then $[\mathbf{G}(\mathbf{x})]^{-1}$ almost surely exists. However, since almost sure convergence does not yield mean-square convergence, the previous result cannot simply be deduced. Finally, one has the following fundamental property (non uniform ellipticity condition replacing the usual uniform ellipticity condition which is not introduced): let $\Omega$ be any bounded open domain of $\mathbb{R}^{d}$ and let $\bar{\Omega}=\Omega \cup \partial \Omega$ be its closure. One then has

$$
E\left\{\left(\sup _{\mathbf{x} \in \bar{\Omega}}\left\|[\mathbf{G}(\mathbf{x})]^{-1}\right\|\right)^{2}\right\}=c_{G}^{2}<+\infty,
$$

in which sup is the supremum and where $0<c_{G}<+\infty$ is a finite positive constant. Note that the mathematical proof of Eq. (32) can easily be derived from (Soize, 2006) for the extended class introduced in Section 3.1 and that Eq. (32) is preoved in (Soize, 2001).

\section{A few properties of the random field $[A]$}

The random field $\mathbf{x} \mapsto[\mathbf{A}(\mathbf{x})]$ indexed by $\Omega$ with values in $M_{6}^{+}(\mathbb{R})$, is defined by Eq. (18) in which the random field $\mathbf{x} \mapsto[\mathbf{G}(\mathbf{x})]$ indexed by $\mathbb{R}^{3}$ with values in $M_{6}^{+}(\mathbb{R})$, is defined in Section 3 with $d=3$ and $n=6$.

\subsection{Basic properties of the random field $[\mathbf{A}]$ and its parameters}

The random field $\mathbf{x} \mapsto[\mathbf{A}(\mathbf{x})]$ is a second-order random field on $\Omega$,

$$
E\left\{\|\mathbf{A}(\mathbf{x})\|^{2}\right\} \leq E\left\{\|\mathbf{A}(\mathbf{x})\|_{F}^{2}\right\}<+\infty
$$

The system of the marginal probability distributions of the random field $\mathbf{x} \mapsto[\mathbf{A}(\mathbf{x})]$ is completely defined, is not Gaussian and is deduced from the system of the marginal probability distributions of the random field $\mathbf{x} \mapsto[\mathbf{G}(\mathbf{x})]$ by using Eq. (18). In general, since $[\underline{a}(\mathbf{x})]$ depends on $\mathbf{x}$, then the random field $\{[\mathbf{A}(\mathbf{x})], \mathbf{x} \in \Omega\}$ is non homogeneous. It can easily be proven that

$$
E\left\{\|[\mathbf{A}(\mathbf{x})]-[\underline{a}(\mathbf{x})]\|_{F}^{2}\right\}=\frac{\delta^{2}}{(n+1)}\left\{\|\underline{a}(\mathbf{x})\|_{F}^{2}+(\operatorname{tr}[\underline{a}(\mathbf{x})])^{2}\right\} .
$$


The dispersion parameter $\delta_{A}(\mathbf{x})$ is such that

$$
\delta_{A}(\mathbf{x})^{2}=\frac{E\left\{\|[\mathbf{A}(\mathbf{x})]-[\underline{a}(\mathbf{x})]\|_{F}^{2}\right\}}{\|\underline{a}(\mathbf{x})\|_{F}^{2}}
$$

and can be rewritten as

$$
\delta_{A}(\mathbf{x})=\frac{\delta}{\sqrt{n+1}}\left\{1+\frac{(\operatorname{tr}[\underline{a}(\mathbf{x})])^{2}}{\operatorname{tr}\left\{[\underline{a}(\mathbf{x})]^{2}\right\}}\right\}^{1 / 2} .
$$

The random field $\mathbf{x} \mapsto[\mathbf{G}(\mathbf{x})]$ almost surely has continuous trajectories (see Section 3.4). If the function $\mathbf{x} \mapsto[\underline{a}(\mathbf{x})]$ is continuous on $\bar{\Omega}$, then the random field $\mathbf{x} \mapsto[\mathbf{A}(\mathbf{x})]$ almost surely has continuous trajectories on $\bar{\Omega}$. Nevertheless, if the function $\mathbf{x} \mapsto[\underline{a}(\mathbf{x})]$ is not continuous on $\bar{\Omega}$, then the random field $\mathbf{x} \mapsto[\mathbf{A}(\mathbf{x})]$ almost surely does not have continuous trajectories on $\bar{\Omega}$.

Then the random field $\mathbf{x} \mapsto[\mathbf{G}(\mathbf{x})]$ is completely and uniquely defined by the following parameters: the $M_{6}^{+}(\mathbb{R})$-valued mean function $\mathbf{x} \mapsto[\underline{a}(\mathbf{x})]$, the positive real parameter $\delta$ and the 63 positive real parameters $L_{1}^{j j^{\prime}}, L_{2}^{j j^{\prime}}, L_{3}^{j j^{\prime}}$ for $1 \leq j \leq j^{\prime} \leq 6$. The smallest number of parameters corresponds to the following case: $\mathbf{x} \mapsto[\underline{a}(\mathbf{x})], \delta$ and $L_{d}=L_{1}^{j j^{\prime}}=L_{2}^{j j^{\prime}}=L_{3}^{j j^{\prime}}$ for all $1 \leq j \leq j^{\prime} \leq 6$.

\subsection{Spatial correlation lengths of the random field $[\mathbf{A}]$ for the homogeneous case}

If $[\underline{a}(\mathbf{x})]=[\underline{a}]$ is independent of $\mathbf{x}$, then the random field $\left\{[\mathbf{A}(\mathbf{x})]=[\underline{L}]^{T}[\mathbf{G}(\mathbf{x})][\underline{L}], \mathbf{x} \in \Omega\right\}$ can be viewed as the restriction to $\Omega$ of a homogeneous random field indexed by $\mathbb{R}^{3}$. Then the dispersion parameter defined by Eq. (35) is independent of $\mathbf{x}$ and then $\delta_{A}(\mathbf{x})=\delta_{A}$. Let $\eta=\left(\eta_{1}, \eta_{2}, \eta_{3}\right) \mapsto r^{A}(\boldsymbol{\eta})$ be the function defined from $\mathbb{R}^{3}$ into $\mathbb{R}$ by

$$
r^{A}(\boldsymbol{\eta})=\frac{\operatorname{tr} E\{([\mathbf{A}(\mathbf{x}+\boldsymbol{\eta})]-[\underline{a}])([\mathbf{A}(\mathbf{x})]-[\underline{a}])\}}{E\left\{\|[\mathbf{A}(\mathbf{x})]-[\underline{a}]\|_{F}^{2}\right\}} .
$$

It can be seen that $r^{A}(0)=1$ and $r^{A}(-\boldsymbol{\eta})=r^{A}(\boldsymbol{\eta})$. For $k=1,2,3$, the spatial correlation length $L_{k}^{A}$ of $\mathbf{x} \mapsto[\mathbf{A}(\mathbf{x})]$ and relative to the coordinate $x_{k}$ can then be defined by

$$
L_{k}^{A}=\int_{0}^{+\infty}\left|r^{A}\left(\eta^{k}\right)\right| d \eta_{k}
$$

in which $\eta^{1}=\left(\eta_{1}, 0,0\right), \eta^{2}=\left(0, \eta_{2}, 0\right)$ and $\eta^{3}=\left(0,0, \eta_{3}\right)$. It is also possible to define a fourth-order tensor $L_{k}^{A_{\alpha \beta \gamma \kappa}}$ of spatial correlation lengths relative to the coordinate $x_{k}$ such that

$$
L_{k}^{A_{\alpha \beta \gamma \kappa}}=\int_{0}^{+\infty}\left|r^{A_{\alpha \beta \gamma \kappa}}\left(\eta^{k}\right)\right| d \eta_{k} \quad, \quad k=1,2,3,
$$

in which

$$
r^{A_{\alpha \beta \gamma \kappa}}(\boldsymbol{\eta})=\frac{1}{\sigma_{\alpha \beta} \sigma_{\gamma \kappa}} E\left\{\left([\mathbf{A}(\mathbf{x}+\boldsymbol{\eta})]_{\alpha \beta}-[\underline{a}]_{\alpha \beta}\right)\left([\mathbf{A}(\mathbf{x})]_{\gamma \kappa}-[\underline{a}]_{\gamma \kappa}\right)\right\},
$$

and where $\sigma_{\alpha \beta}=\sqrt{E\left\{\left([\mathbf{A}(\mathbf{x})]_{\alpha \beta}-[\underline{a}]_{\alpha \beta}\right)^{2}\right\}}$. 


\section{Discretizing with stochastic finite elements and solving the random equation}

For all $\ell$ and $m$ in $\{1,2,3\}$, one must

(1) solve the stochastic BVP defined by Eqs. (9)-(10) with the random constitutive equation $\boldsymbol{\sigma}^{\ell m}(\mathbf{x})=\mathbb{C}(\mathbf{x}): \varepsilon\left(\mathbf{D}^{\ell m}(\mathbf{x})\right)$ in order to construct the random local displacement field $\mathbf{D}^{\ell m}$.

(2) calculate the fourth-order tensor-valued random field $H$ defined by Eq. (11) and corresponding to the strain localization.

(3) estimate the probabilistic properties of the fourth-order random effective stiffness tensor $\mathbb{C}^{\text {eff }}$ defined by Eq. (14).

In order to solve this problem, the following computational stochastic method is used.

(1) The weak formulation of the stochastic BVP is constructed and the existence of a unique second-order stochastic solution is proven.

(2) The stochastic finite element method is used for discretizing the weak formulation and Eqs. (11) and (14). For such a numerical approximation, the random local stiffness tensor field $\mathbf{x} \mapsto \mathbb{C}_{j k \ell m}(\mathbf{x})$ and the random local strain tensor field $\mathbf{x} \mapsto \varepsilon_{j k}\left(\mathbf{D}^{\ell m}(\mathbf{x})\right)$ are discretized at all the Gauss-Legendre quadrature points of the finite elements.

(3) The probabilistic quantities are then estimated by using the Monte Carlo numerical simulation method which is made up of 3 main steps: (a) developing a generator for constructing $n_{s}$ independent realizations $\left\{\mathbf{x} \mapsto \mathbb{C}\left(\mathbf{x}, \theta_{r}\right), r=1, \ldots n_{s}\right\}$ of the random field $\mathbf{x} \mapsto \mathbb{C}(\mathbf{x})$ using the probability model presented in Sections 3 and 4; (b) for each realization $\mathbf{x} \mapsto \mathbb{C}\left(\mathbf{x}, \theta_{r}\right)$, calculating the corresponding realization $\mathbb{C}^{\text {eff }}\left(\theta_{r}\right)$ of the effective tensor (and related quantities such as the random eigenvalues of the random tensor $\mathbb{C}^{\text {eff }}$ ) by solving a deterministic matrix equation; (c) with the $n_{s}$ independent realizations, estimating the probabilistic quantities (moments, probability distributions) using the mathematical statistics and studying the convergence with respect to $n_{s}$.

\subsection{Weak formulation of the stochastic BVP}

Below, $\ell$ and $m$ are fixed in $\{1,2,3\}$. Let $\mathbf{x} \mapsto \mathbf{d}(\mathbf{x})=\left(d_{1}(\mathbf{x}), d_{2}(\mathbf{x}), d_{3}(\mathbf{x})\right)$ be a function from $\Omega$ into $\mathbb{R}^{3}$. Let $V=\left(H^{1}(\Omega)\right)^{3}, V_{0} \subset V$ and $V_{\ell m} \subset V$ be the real Hilbert spaces such that

$$
\begin{gathered}
V=\left\{d_{j} \in L^{2}(\Omega) \quad, \quad \frac{\partial d_{j}}{\partial x_{k}} \in L^{2}(\Omega) \quad, \quad \text { for } j \text { and } k=1,2,3\right\} \\
V_{0}=\{\mathbf{d} \in V, \mathbf{d}(\mathbf{x})=0 \quad \text { for } \mathbf{x} \in \partial \Omega\} \quad, \\
V_{\ell m}=\left\{\mathbf{d} \in V, \quad \mathbf{d}(\mathbf{x})=\mathbf{d}^{\ell m}(\mathbf{x}) \text { for } \mathbf{x} \in \partial \Omega\right\}
\end{gathered}
$$

in which $\mathbf{d}^{\ell m}$ is the function from $\partial \Omega$ into $\mathbb{R}^{3}$ such that $\mathbf{d}^{\ell m}(\mathbf{x})=g^{\ell m} \mathbf{x}$. The inner product in $V$ is denoted by $\left\langle\mathbf{d}, \mathbf{d}^{\prime}\right\rangle_{V}$ and the associated norm is denoted by $\|\mathbf{d}\|_{V}$. Let $\mathbb{V}, \mathbb{V}_{0} \subset \mathbb{V}$ and $\mathbb{V}_{\ell m} \subset \mathbb{V}$ be the three real Hilbert spaces of all the second-order random variables $\theta \mapsto\{\mathbf{x} \mapsto \mathbf{D}(\mathbf{x}, \theta)\}$ defined on probability space $(\Theta, \mathcal{T}, P)$, with values in $V, V_{0}$ and $V_{\ell m}$ respectively,

$$
\mathbb{V}=L^{2}(\Theta, V) \quad, \quad \mathbb{V}_{0}=L^{2}\left(\Theta, V_{0}\right) \quad, \quad \mathbb{V}_{\ell m}=L^{2}\left(\Theta, V_{\ell m}\right)
$$

The inner product in $\mathbb{V}$ and the associated norm are such that

$$
\ll \mathbf{D}, \delta \mathbf{D} \gg_{\mathbb{V}}=E\left\{<\mathbf{D}, \delta \mathbf{D}>_{V}\right\} \quad \text { and } \quad\|\mathbf{D}\|_{\mathbb{V}}=\left(E\left\{\|\mathbf{D}\|_{V}^{2}\right\}\right)^{1 / 2}
$$


The new indices $I=(j, k)$ and $J=(\ell, m)$ introduced in Section 2.3 are used and the strain vector

$$
\mathbf{e}(\mathbf{d})=\left(\varepsilon_{11}(\mathbf{d}), \varepsilon_{22}(\mathbf{d}), \varepsilon_{33}(\mathbf{d}), 2 \varepsilon_{12}(\mathbf{d}), 2 \varepsilon_{13}(\mathbf{d}), 2 \varepsilon_{23}(\mathbf{d})\right)
$$

is introduced. The weak formulation of the stochastic BVP defined by Eqs. (9)-(10) can then be written as follows. Find the random field $\mathbf{D}^{\ell m}$ in $\mathbb{V}_{\ell m}$ such that,

$$
\mathbf{K}\left(\mathbf{D}^{\ell m}, \delta \mathbf{D}\right)=0 \quad \text { a.s. } \quad, \quad \forall \delta \mathbf{D} \in \mathbb{V}_{0},
$$

in which the random bilinear form $(\mathbf{D}, \delta \mathbf{D}) \mapsto \mathbf{K}(\mathbf{D}, \delta \mathbf{D})$ on $\mathbb{V} \times \mathbb{V}$ is defined by

$$
\mathbf{K}(\mathbf{D}, \delta \mathbf{D})=\int_{\Omega}<[\mathbf{A}(\mathbf{x})] \mathbf{e}(\mathbf{D}(\mathbf{x})), \mathbf{e}(\delta \mathbf{D}(\mathbf{x}))>d \mathbf{x}
$$

\subsection{Existence and uniqueness of a second-order solution}

The following problem: find $\mathbf{D}^{\ell m}$ in $\mathbb{V}_{\ell m}$ such that, $\mathbf{K}\left(\mathbf{D}^{\ell m}, \delta \mathbf{D}\right)=0$ a.s. for all $\delta \mathbf{D}$ in $\mathbb{V}_{0}$, has a unique solution.

To prove this result and in order to simplify the notation, the random field $\mathbf{D}^{\ell m}$ is rewritten as $\mathbf{D}$ (no confusion is possible).

(i) Due to the fundamental property defined by Eq. (32) (non uniform ellipticity condition replacing the usual uniform ellipticity condition), it is proven (Soize, 2006) that, for all random field $\mathbf{D}$ in $\mathbb{V}$ such that $\mathbf{D}$ is not a random rigid body displacement field, one has

$$
\sqrt{E\left\{\mathbf{K}(\mathbf{D}, \mathbf{D})^{2}\right\}} \geq c_{K}\|\mathbf{D}\|_{\mathbb{V}}^{2}
$$

in which $c_{K}$ is a finite positive real constant.

(ii) The usual proof of the existence of a solution can be directly deduced from Eq. (40) if a uniform ellipticity condition and a uniform boundness condition for the fourth-order tensorvalued random field $\mathbf{x} \mapsto \mathbb{C}(\mathbf{x})$ is used. This usual proof cannot be used here due to the non introduction of these uniformness conditions which are substituted by the more realistic probabilistic hypothesis which has been introduced and which corresponds to the introduction of the non uniformness condition. Therefore, the weak formulation is reformulated using a deterministic Lagrange multiplier field $\mathbf{x} \mapsto \mathbf{q}(\mathbf{x})$ from $\partial \Omega$ in $\mathbb{R}^{3}$ belonging to the space $Q_{\partial \Omega}$ which is defined as the continuous dual space of the space $V_{\partial \Omega}$ constituted of all the functions $\mathbf{x} \mapsto \mathbf{d}_{\mid \partial \Omega}(\mathbf{x})$ from $\partial \Omega$ in $\mathbb{R}^{3}$ which are the traces on $\partial \Omega$ of the functions $\mathbf{d}$ belonging to $V$. Find $\mathbf{D}$ in $\mathbb{V}$ and $\mathbf{q}$ in $Q_{\partial \Omega}$ such that, for all $\delta \mathbf{D}$ in $\mathbb{V}$ and for all $\delta \mathbf{q}$ in $Q_{\partial \Omega}$, one has

$$
\mathbf{K}(\mathbf{D}, \delta \mathbf{D})+\ll \delta \mathbf{q}, \mathbf{D}_{\left.\right|_{\partial \Omega}}-\mathbf{d}^{\ell m} \gg+\ll \mathbf{q}, \delta \mathbf{D}_{\left.\right|_{\partial \Omega}} \gg=0 \text { a.s. }
$$

in which $\mathbf{d}^{\ell m}$ is the function from $\partial \Omega$ into $\mathbb{R}^{3}$ defined in Section 5.1 and where $\ll \mathbf{q}, \mathbf{v} \gg$ is the dual bracket between $Q_{\partial \Omega}$ and $V_{\partial \Omega}$.

(iii) One now proves the existence of a solution $\mathbf{D}$ in $\mathbb{V}_{\ell m}$ of Eq. (43).

(iii-1) If $\mathbf{D}$ is a random rigid body displacement field, then $\mathbf{K}(\mathbf{D}, \delta \mathbf{D})=0$ and consequently, Eq. (43) shows that, for all $\delta \mathbf{D}$ in $\mathbb{V}$ and for all $\delta \mathbf{q}$ in $Q_{\partial \Omega}$, one has $\ll \delta \mathbf{q}, \mathbf{D}_{\mid \partial \Omega}-\mathbf{d}^{\ell m} \gg+\ll$ $\mathbf{q}, \delta \mathbf{D}_{\mid \partial \Omega} \gg=0$ a.s.. This equation yields $\mathbf{D}_{\left.\right|_{\partial \Omega}}=\mathbf{d}^{\ell m}$ which contracdicts with the fact that $\mathbf{d}^{\ell m}$ is not the trace on $\partial \Omega$ of a rigid body displacement field. 
(iii-2) It is now assumed that $\mathbf{D}$ belongs to $\mathbb{V}$ but is not a random rigid body displacement field. In this condition, Eq. (42) holds. Taking $\delta \mathbf{D}=\mathbf{D}$ and $\delta \mathbf{q}=\mathbf{q} \in Q_{\partial \Omega}$ in Eq. (43) yields

$$
\mathbf{K}(\mathbf{D}, \mathbf{D})+\ll \mathbf{q}, 2 \mathbf{D}_{\mid \partial \Omega}-\mathbf{d}^{\ell m} \gg=0 \text { a.s. } .
$$

Equations (42) and (44) yield

$$
c_{K}^{2}\|\mathbf{D}\|_{\mathbb{V}}^{4} \leq E\left\{\ll \mathbf{q}, 2 \mathbf{D}_{\left.\right|_{\partial \Omega}}-\mathbf{d}^{\ell m} \gg^{2}\right\}
$$

It can easily be proven that

$$
E\left\{\ll \mathbf{q}, 2 \mathbf{D}_{\mid \partial \Omega}-\mathbf{d}^{\ell m} \gg^{2}\right\} \leq c_{1}+c_{2}\|\mathbf{D}\|_{\mathbb{V}}^{2}
$$

in which $c_{1}=2\|\mathbf{q}\|_{Q_{\partial \Omega}}^{2}\left\|\mathbf{d}^{\ell m}\right\|_{V_{\partial \Omega}}^{2}<+\infty$ and $c_{2}=8 c_{\gamma}^{2}\|\mathbf{q}\|_{Q_{\partial \Omega}}^{2}<+\infty$ in which $c_{\gamma}$ is the finite constant such that $\|\mathbf{d}\|_{V_{\partial \Omega}} \leq c_{\gamma}\|\mathbf{d}\|_{V}$. From Eqs. (45) and (46), it can be deduced that $c_{K}^{2}\|\mathbf{D}\|_{\vee}^{4} \leq c_{1}+c_{2}\|\mathbf{D}\|_{\mathbb{V}}^{2}$ which yields $\|\mathbf{D}\|_{\vee} \leq c<+\infty$. One then has proven the existence of a solution in $\mathbb{V}$ but since any solution of Eq. (43) is such that $\mathbf{D}_{\left.\right|_{\partial \Omega}}=\mathbf{d}^{\ell m}$ on $\partial \Omega$, one has proven the existence of a solution $\mathbf{D}$ in $\mathbb{V}_{\ell m}$.

(iv) The proof of the uniqueness is straightforward.

\subsection{Finite element discretization}

The stochastic finite element method is used to discretize the weak formulation defined by Eq. (40).

(i) A finite element mesh of domain $\Omega$ is carried out using 3D solid finite elements. One then has $\Omega=\cup_{e} \Omega_{e}$ in which $\Omega_{e}$ is the domain of the finite element number $e$. Any displacement field $\mathbf{x} \mapsto \mathbf{d}(\mathbf{x})$ in $V$ and its associated strain vector field $\mathbf{x} \mapsto \mathbf{e}(\mathbf{x})$ are then approximated by

$$
\mathbf{d}(\mathbf{x}) \simeq[B(\mathbf{x})] \mathbf{w}, \mathbf{x} \in \bar{\Omega}, \mathbf{e}(\mathbf{x}) \simeq[S(\mathbf{x})] \mathbf{w}, \mathbf{x} \in \Omega
$$

in which $\mathbf{w}=\left(w_{1}, \ldots, w_{\nu}\right)$ is the vector of the $\nu$ degrees of freedom corresponding to the values of the components of the field $\mathbf{d}$ at the nodes of the mesh. The $(3 \times \nu)$ real matrices $[B(\mathbf{x})]$ and $[S(\mathbf{x})]$ are known matrices usually constructed by using the interpolation functions of the finite elements. For any integrable function $\mathbf{x} \mapsto f(\mathbf{x})$ defined on $\Omega$ and continuous on $\Omega_{e}$, the following usual numerical approximation can be written

$$
\int_{\Omega_{e}} f(\mathbf{x}) d \mathbf{x} \simeq \sum_{k=1}^{N_{e}} \omega_{\alpha_{k}} f\left(\mathbf{x}^{\alpha_{k}}\right)
$$

in which $\left\{\omega_{\alpha_{1}}, \ldots, \omega_{\alpha_{N_{e}}}\right\}$ and $\left\{\mathbf{x}^{\alpha_{1}}, \ldots, \mathbf{x}^{\alpha_{N_{e}}}\right\}$ are the sets of all the $N_{e}$ weights and the $N_{e}$ Gauss-Legendre quadrature points for the finite element $\Omega_{e}$. Consequently, it can be written that

$$
\int_{\Omega} f(\mathbf{x}) d \mathbf{x}=\sum_{e} \int_{\Omega_{e}} f(\mathbf{x}) d \mathbf{x} \simeq \sum_{\alpha=1}^{N} \omega_{\alpha} f\left(\mathbf{x}^{\alpha}\right)
$$

in which $\left\{\omega_{1}, \ldots, \omega_{N}\right\}$ and $\left\{\mathbf{x}^{1}, \ldots, \mathbf{x}^{N}\right\}$ are the sets of all the $N$ weights and the $N$ GaussLegendre quadrature points for $\Omega=\cup_{e} \Omega_{e}$ with $N=\sum_{e} N_{e}$. 
For all $\mathbf{x}$ in $\bar{\Omega}$, the finite element approximation of all $\mathbf{D}$ and $\delta \mathbf{D}$ in $\mathbb{V}$ is then written as $\mathbf{D}(\mathbf{x}) \simeq$ $[B(\mathbf{x})] \mathbf{W}$ and $\delta \mathbf{D}(\mathbf{x}) \simeq[B(\mathbf{x})] \delta \mathbf{W}$, in which $\mathbf{W}$ and $\delta \mathbf{W}$ are $\mathbb{R}^{\nu}$-valued second-order random vectors. Therefore, the corresponding finite element approximation of the random bilinear form defined by Eq. (41) is such that

$$
<[\mathbf{K}] \mathbf{W}, \delta \mathbf{W}>=\mathbf{K}([B(.)] \mathbf{W},[B(.)] \delta \mathbf{W})
$$

defining the $(\nu \times \nu)$ random stiffness matrix $[\mathbf{K}]$ such that

$$
[\mathbf{K}]=\int_{\Omega}[S(\mathbf{x})]^{T}[\mathbf{A}(\mathbf{x})][S(\mathbf{x})]
$$

(ii) Using Eq. (47), the random stiffness matrix $[\mathbf{K}]$ defined by Eq. (48) can be approximated by the random matrix $\left[\mathbf{K}_{N}\right]$ such that

$$
\left[\mathbf{K}_{N}\right]=\sum_{\alpha=1}^{N} \omega_{\alpha}\left[S\left(\mathbf{x}^{\alpha}\right)\right]^{T}\left[\mathbf{A}\left(\mathbf{x}^{\alpha}\right)\right]\left[S\left(\mathbf{x}^{\alpha}\right)\right] d \mathbf{x}
$$

in which the $M_{6}^{+}(\mathbb{R})$-valued random field $\mathbf{x} \mapsto[\mathbf{A}(\mathbf{x})]$ is discretized in the $N$ Gauss-Legendre quadrature points $\mathbf{x}^{1}, \ldots, \mathbf{x}^{N}$. In order to analyse the value of $N$ which is required to obtain a good approximation of $[\mathbf{K}]$ by $\left[\mathbf{K}_{N}\right]$ and taking into account Section 5.2, it can be deduced that the integral over $\Omega$ in Eq. (48) must be read as a mean-square integral. In the vector space of all the second-order random matrices with values in $M_{6}(\mathbb{R})$, a necessary and sufficient condition (Krée and Soize, 1986) for that the sequence of second-order random matrices $\left\{\left[\mathbf{K}_{N}\right]\right\}_{N}$ converges to the second-order random matrix $[\mathbf{K}]$ when $N$ goes to infinity is

$$
\lim _{N, N^{\prime} \rightarrow+\infty} E\left\{\operatorname{tr}\left(\left[\mathbf{K}_{N}\right]^{T}\left[\mathbf{K}_{N^{\prime}}\right]\right)\right\}=E\left\{\operatorname{tr}\left([\mathbf{K}]^{T}[\mathbf{K}]\right)\right\}
$$

Since $[\mathbf{K}]$ and $\left[\mathbf{K}_{N}\right]$ are symmetric matrices, it can be deduced that $\left[\mathbf{K}_{N}\right]$ will be a good approximation of $[\mathbf{K}]$ in the mean-square sense if $E\left\{\operatorname{tr}\left(\left[\mathbf{K}_{N}\right]^{2}\right)\right\} \simeq E\left\{\operatorname{tr}\left([\mathbf{K}]^{2}\right)\right\}$. One has

$$
\begin{gathered}
E\left\{\operatorname{tr}\left([\mathbf{K}]^{2}\right)\right\}=\int_{\Omega} \int_{\Omega} \sum_{k, \ell, k^{\prime}, \ell^{\prime}} \mathcal{S}_{k \ell k^{\prime} \ell^{\prime}}(\mathbf{x}, \mathbf{y}) R_{k \ell k^{\prime} \ell^{\prime}}(\mathbf{x}, \mathbf{y}) d \mathbf{x} d \mathbf{y} \\
E\left\{\operatorname{tr}\left(\left[\mathbf{K}_{N}\right]^{2}\right)\right\}=\sum_{\alpha, \beta=1}^{N} \omega_{\alpha} \omega_{\beta} \sum_{k, \ell, k^{\prime}, \ell^{\prime}} \mathcal{S}_{k \ell k^{\prime} \ell^{\prime}}\left(\mathbf{x}^{\alpha}, \mathbf{x}^{\beta}\right) R_{k \ell k^{\prime} \ell^{\prime}}\left(\mathbf{x}^{\alpha}, \mathbf{x}^{\beta}\right)
\end{gathered}
$$

in which

$$
\begin{gathered}
\mathcal{S}_{k \ell k^{\prime} \ell^{\prime}}(\mathbf{x}, \mathbf{y})=\sum_{i, j=1}^{\nu}[S(\mathbf{x})]_{k i}[S(\mathbf{x})]_{\ell j}[S(\mathbf{y})]_{k^{\prime} i}[S(\mathbf{y})]_{\ell^{\prime} j}, \\
R_{k \ell k^{\prime} \ell^{\prime}}(\mathbf{x}, \mathbf{y})=E\left\{[\mathbf{A}(\mathbf{x})]_{k \ell}[\mathbf{A}(\mathbf{y})]_{k^{\prime} \ell^{\prime}}\right\} .
\end{gathered}
$$

Thus the convergence is clearly reached when $N$ goes to infinity. For a given fixed $N$, the random matrix $\left[\mathbf{K}_{N}\right]$ will be a good approximation of the random matrix $[\mathbf{K}]$ in the mean-square sense if the number $N$ of the Gauss-Legendre quadrature points $\mathbf{x}^{1}, \ldots, \mathbf{x}^{N}$ is sufficiently large and is adapted to the variations of the intercorrelation functions $R_{k \ell k^{\prime} \ell^{\prime}}$ of the real-valued random fields $[\mathbf{A}]_{k \ell}$ and $[\mathbf{A}]_{k^{\prime} \ell^{\prime}}$. 
(iii) The finite element discretization of the random field $\mathbf{D}^{\ell m}$ in $\mathbb{V}_{\ell m}$ which is solution of Eq. (40) is then written as

$$
\mathbf{D}^{\ell m}(\mathbf{x}) \simeq[B(\mathbf{x})] \mathbf{W}^{\ell m} \quad, \quad \mathbf{x} \in \bar{\Omega} .
$$

The second-order random vector $\mathbf{W}^{\ell m}$ with values in $\mathbb{R}^{\nu}$ can then be written as

$$
\mathbf{W}^{\ell m}=\left(\mathbf{W}_{i}^{\ell m}, \mathbf{w}_{b}^{\ell m}\right)
$$

in which $\mathbf{W}_{i}^{\ell m}$ is the $\mathbb{R}^{\nu_{i}}$-valued second-order random vector of the $\nu_{i}$ degrees of freedom for the nodes inside the domain $\Omega$ and where $\mathbf{w}_{b}^{\ell m}$ is the $\mathbb{R}^{\nu_{b}}$ deterministic vector of the $\nu_{b}$ degrees of freedom for the nodes belonging to the boundary $\partial \Omega$. This last vector is such that $\mathbf{d}^{\ell m}(\mathbf{x}) \simeq[B(\mathbf{x})] \mathbf{w}_{b}^{\ell m}$ for all $\mathbf{x}$ in $\partial \Omega$ and the vector $\mathbf{w}_{b}^{\ell m}$ is constituted of the values of the components of the field $\mathbf{d}^{\ell m}$ at the nodes belonging to boundary $\partial \Omega$. The block writing of the random stiffness matrix $[\mathbf{K}]$ related to Eq. (50) is introduced as follows,

$$
[\mathbf{K}]=\left[\begin{array}{cc}
{\left[\mathbf{K}_{i i}\right]} & {\left[\mathbf{K}_{i b}\right]} \\
{\left[\mathbf{K}_{i b}\right]^{T}} & {\left[\mathbf{K}_{b b}\right]}
\end{array}\right] .
$$

The finite element approximation of Eq. (40) is then given by the following random matrix equation allowing the unknown random vector $\mathbf{W}_{i}^{\ell m}$ to be calculated,

$$
\left[\mathbf{K}_{i i}\right] \mathbf{W}_{i}^{\ell m}=-\left[\mathbf{K}_{i b}\right] \mathbf{w}_{b}^{\ell m}
$$

The random values $H\left(\mathbf{x}^{1}\right), \ldots, \leftrightarrow\left(\mathbf{x}^{N}\right)$ of $H$ at the $N$ points $\mathbf{x}^{1}, \ldots, \mathbf{x}^{N}$ are calculated by using Eq. (11). Let $\mathbb{C}\left(\mathbf{x}^{1}\right), \ldots, \mathbb{C}\left(\mathbf{x}^{N}\right)$ be the random values of $\mathbb{C}$ at the $N$ points $\mathbf{x}^{1}, \ldots, \mathbf{x}^{N}$. Then, using Eq. (47), the finite element approximation of the fourth-order random effective stiffness tensor $\mathbb{C}^{\text {eff }}$ defined by Eq. (14) can be written as

$$
\mathbb{C}^{\mathrm{eff}} \simeq \frac{1}{|\Omega|} \sum_{\alpha=1}^{N} \omega_{\alpha} \mathbb{C}\left(\mathbf{x}^{\alpha}\right): \mathbb{H}\left(\mathbf{x}^{\alpha}\right)
$$

Let $\left[\mathbf{A}^{\text {eff }}\right]$ be the $M_{6}^{+}(\mathbb{R})$-valued random matrix of the fourth-order random effective stiffness tensor $\mathbb{C}^{\text {eff }}$ such that

$$
\left[\mathbf{A}^{\mathrm{eff}}\right]_{I J}=\mathbb{C}_{j k \ell m}^{\mathrm{eff}}
$$

In order to perform the probability analysis of the fourth-order random effective stiffness tensor, one introduces the following order statistics

$$
\widetilde{\Lambda}_{1} \geq \widetilde{\Lambda}_{2} \geq \ldots \geq \widetilde{\Lambda}_{6}>0
$$

of the random eigenvalues $\widetilde{\Lambda}$ of the random matrix $\left[\mathbf{A}^{\text {eff }}\right]$ such that

$$
\left[\mathbf{A}^{\mathrm{eff}}\right] \boldsymbol{\Phi}=\widetilde{\Lambda} \boldsymbol{\Phi}
$$

The random variable $\left\|\mathbf{A}^{\text {eff }}\right\|$ defined by Eq. (30) is such that

$$
\left\|\mathbf{A}^{\mathrm{eff}}\right\|=\widetilde{\Lambda}_{1}
$$




\subsection{Defining the probabilistic quantities for the random effective stiffness matrix}

Taking into account the large quantity of probabilistic information which can be constructed, it is necessary to limit the quantities which will be computed.

In this context, it is assumed that the mean model of the microstructure is homogeneous, that is to say $[\underline{a}(\mathbf{x})]=[\underline{a}]$ is independent of $\mathbf{x}$. Consequently (see Section 4.2), the random field $[\mathbf{A}]$ describing the fourth-order tensor-valued random field $\mathbb{C}$ of the random anisotropic microstructure can be viewed as the restriction to $\Omega$ of a homogeneous random field indexed by $\mathbb{R}^{3}$. The dispersion parameter $\delta_{A}$ defined by Eq. (36) is then independent of $\mathbf{x}$ and can easily be deduced from the value of the dispersion parameter $\delta$ defined by Eq. (27). Finally, the spatial correlation lengths $L_{1}^{A}, L_{2}^{A}$ and $L_{3}^{A}$ defined by Eq. (38) depend on the values of the parameters $L_{1}^{j j^{\prime}}, L_{2}^{j j^{\prime}}, L_{3}^{j j^{\prime}}$ for $1 \leq j \leq j^{\prime} \leq 6$ of the stochastic germs (see Section 3.1).

Therefore, every probabilistic analysis of the macroscopic properties of the random anisotropic elastic microstructure is performed for a given value of the parameters $[\underline{a}], \delta$ and $L_{1}^{j j^{\prime}}, L_{2}^{j j^{\prime}}, L_{3}^{j j^{\prime}}$ for $1 \leq j \leq j^{\prime} \leq 6$.

The following probabilistic quantities can be constructed.

(i) The mean value $\left[m_{\mathbf{A}^{\text {eff }}}\right]_{I J}$, the standard deviation $\left[\sigma_{\mathbf{A}^{\text {eff }}}\right]_{I J}$ and the coefficient of variation $\left[\mathrm{cv}_{\mathbf{A}^{\mathrm{eff}}}\right]_{I J}$ of the components $\left[\mathbf{A}^{\mathrm{eff}}\right]_{I J}$ of the random effective stiffness matrix $\left[\mathbf{A}^{\mathrm{eff}}\right]$ are such that

$$
\begin{gathered}
{\left[m_{\mathbf{A}^{\mathrm{eff}}}\right]_{I J}=E\left\{\left[\mathbf{A}^{\mathrm{eff}}\right]_{I J}\right\},} \\
{\left[\sigma_{\mathbf{A}^{\mathrm{eff}}}\right]_{I J}=\sqrt{E\left\{\left(\left[\mathbf{A}^{\mathrm{eff}}\right]_{I J}-\left[m_{\mathbf{A}^{\mathrm{eff}}}\right]_{I J}\right)^{2}\right\}},} \\
{\left[\mathbf{c v}_{\mathbf{A}^{\mathrm{eff}}}\right]_{I J}=\frac{\left[\sigma_{\mathbf{A}^{\mathrm{eff}}}\right]_{I J}}{\left[m_{\mathbf{A}^{\mathrm{eff}}}\right]_{I J}} .}
\end{gathered}
$$

(ii) The mean value $m_{\left\|\mathbf{A}^{\text {eff }}\right\|}$, the standard deviation $\sigma_{\left\|\mathbf{A}^{\text {eff }}\right\|}$ and the coefficient of variation $\mathrm{cv}_{\left\|\mathbf{A}^{\text {eff }}\right\|}$ of the random variable $\left\|\mathbf{A}^{\text {eff }}\right\|$ are given by

$$
\begin{gathered}
m_{\| \mathbf{A}^{\text {eff } \|}}=E\left\{\left\|\mathbf{A}^{\text {eff }}\right\|\right\}, \\
\sigma_{\left\|\mathbf{A}_{\text {eff }}\right\|}=\sqrt{E\left\{\left(\left\|\mathbf{A}^{\text {eff }}\right\|-m_{\left\|\mathbf{A}^{\text {eff }}\right\|}\right)^{2}\right\}}, \\
\mathrm{cv}_{\| \mathbf{A}^{\text {eff } \|}}=\frac{\sigma_{\left\|\mathbf{A}^{\text {eff }}\right\|}}{m_{\left\|\mathbf{A}^{\text {eff }}\right\|}} .
\end{gathered}
$$

The Tchebychev inequality for the positive-valued random variable $\left\|\mathbf{A}^{\text {eff }}\right\|$ can be written as

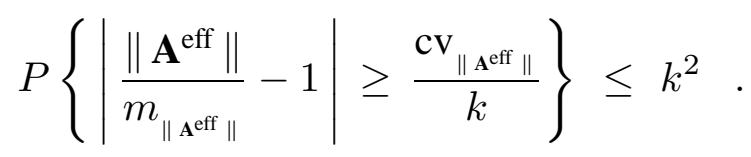

From this inequality, it can be deduced that $\left\|\mathbf{A}^{\text {eff }}\right\|$ converges in probability to the mean value $m_{\left\|\mathbf{A}^{\text {eff }}\right\|}$ when $\mathrm{cv}_{\left\|\mathbf{A}_{\text {eff }}\right\|}$ goes to zero. Consequently, the study of $\mathrm{cv}_{\left\|\mathbf{A}^{\text {eff }}\right\|}$ defined by Eq. (63), as a function of the spatial correlation lengths (or equivalently, as a function of the RVE size), allows the statistic fluctuations of the random matrix $\left[\mathbf{A}^{\text {eff }}\right]$ to be analysed. Such a quantification can be improved in constructing the cumulative probability distribution defined in point (iii) below.

(iii) Let $z \mapsto p_{Z}(z)$ be the probability density function with respect to $d z$ of the random variable $Z$ defined by

$$
Z=\frac{\left\|\mathbf{A}^{\text {eff }}\right\|}{m_{\left\|\mathbf{A}^{\text {eff }}\right\|}} .
$$


The support of $p_{Z}$ is $\mathbb{R}^{+}$. The cumulative distribution function $z^{*} \mapsto F_{Z}\left(z^{*}\right)$ of the random variable $Z$ is then defined by

$$
F_{Z}\left(z^{*}\right)=\int_{0}^{z^{*}} p_{Z}(z) d z=E\left\{\mathbb{1}_{Z \leq z^{*}}\right\}
$$

in which $\mathbb{1}_{Z \leq z^{*}}=1$ if $Z \leq z^{*}$ and $=0$ if not.

\subsection{Solving the random equation and computing the statistical estimations}

The random equations (52) to (57) are solved by the Monte Carlo numerical simulation method by using $n_{s}$ independent realizations $\theta_{1}, \ldots, \theta_{n_{s}}$ in $\Theta$.

(i) The independent stochastic germs $U_{j j^{\prime}}$ for $1 \leq j \leq j^{\prime} \leq 6$ are simulated in the $N$ GaussLegendre quadrature points $\mathbf{x}^{1}, \ldots, \mathbf{x}^{N}$ (see Section 5.3). Let $U$ be any one of the random fields $U_{j j^{\prime}}$ for fixed $j$ and $j^{\prime}$. One then has to simulate realizations of the random vector $\mathbf{U}=\left(U\left(\mathbf{x}^{1}\right), \ldots, U\left(\mathbf{x}^{N}\right)\right)$. A first representation adapted to a large value of $N$ is based on the usual numerical simulation of homogeneous Gaussian vector-valued random field $U$ constructed with the stochastic integral representation of homogeneous stochastic fields (see for instance (Shinozuka, 1971), (Poirion and Soize, 1995)). A second representation adapted to a small or moderate value of $N$ consists in writing $\mathbf{U}=\left[L_{\mathbf{U}}\right]^{T} \boldsymbol{\eta}$ in which $\eta=\left(\eta_{1}, \ldots, \eta_{N}\right)$ is an $\mathbb{R}^{N_{-}}$ valued random variable whose components $\eta_{1}, \ldots, \eta_{N}$ are $N$ independent normalized Gaussian random variables $\left(E\left\{\eta_{j}\right\}=0\right.$ and $E\left\{\eta_{j}^{2}\right\}=1$ for $\left.j=1, \ldots, N\right)$ and where $\left[L_{\mathbf{U}}\right]$ is the upper real triangular matrix corresponding to the Cholesky factorization $\left[C_{\mathbf{U}}\right]=\left[L_{\mathbf{U}}\right]^{T}\left[L_{\mathbf{U}}\right]$ of the covariance matrix $\left[C_{\mathbf{U}}\right]$ in $M_{N}^{+}(\mathbb{R})$ such that $\left[C_{\mathbf{U}}\right]_{i j}=R_{U}\left(\mathbf{x}^{i}-\mathbf{x}^{j}\right)$. A third representation also adapted to a large value of $N$ consists in projecting $\mathbf{U}$ in the dominant eigensubspace of $\left[C_{\mathbf{U}}\right]$. This dominant eigensubspace is constructed with the eigenvectors of $\left[C_{\mathbf{U}}\right]$ associated with the first largest eigenvalues. The eigenvalue problem is solved with an iterative method (such as the Lanczos or the subspace iteration method) without effective assemblage of matrix $\left[C_{\mathbf{U}}\right]$ (see Soize, 2006).

(ii) The mathematical expectation of any random quantity $R$ in Eqs. (58) to (63) is estimated by

$$
E\{R\}=\frac{1}{n_{s}} \sum_{r=1}^{n_{s}} R\left(\theta_{r}\right)
$$

The cumulative probability distribution $F_{Z}$ defined by Eq. (66) is estimated with the usual estimator (Serfling, 1980).

Let $\nu$ be the number of degrees of freedom of the finite element model introduced in Section 5.3. Let $\left[\mathbf{A}_{\nu}^{\text {eff }}\right]$ be the random effective stiffness matrix calculated with the finite element model having $\nu$ degrees of freedom. For a given value of $\nu$, the convergence of the Monte Carlo numerical simulation with respect to the number $n_{s}$ of realizations can be controlled by studying the function

$$
n_{s} \mapsto \operatorname{conv}\left(n_{s}, \nu\right)=\left(\frac{1}{n_{s}} \sum_{r=1}^{n_{s}}\left\|\mathbf{A}_{\nu}^{\text {eff }}\left(\theta_{r}\right)\right\|^{2}\right)^{1 / 2} /\left\|\underline{\mathbf{A}}_{\nu}^{\text {eff }}\right\|
$$

in which $\left\|\mathbf{A}_{\nu}^{\text {eff }}\left(\theta_{r}\right)\right\|$ is the realization $\theta_{r}$ of the random variable $\left\|\mathbf{A}_{\nu}^{\text {eff }}\right\|$ defined by Eq. (30) in which the random effective stiffness matrix $\left[\mathbf{A}_{\nu}^{\text {eff }}\right]$ is defined by Eq. (57) and where $\left\|\underline{\mathbf{A}}_{\nu}^{\text {eff }}\right\|$ is the norm defined by Eq. (30) of the effective stiffness matrix $\left[\underline{\mathbf{A}}_{\nu}^{\mathrm{eff}}\right]$ of the mean model. If the mean model of the microstructure is homogeneous, then the local stiffness matrix $[\underline{a}]$ is independent of $\mathbf{x}$ and then $\left[\underline{\mathbf{A}}_{\nu}^{\text {eff }}\right]=[\underline{a}]$. The right-hand side of Eq. (68) corresponds to the statistical estimation 
of the norm $\left\|\left|\mathbf{A}_{\nu}^{\text {eff }}\right|\right\|=\left(E\left\{\left\|\mathbf{A}_{\nu}^{\text {eff }}\right\|^{2}\right\}\right)^{1 / 2}$ of the random matrix $\left[\mathbf{A}_{\nu}^{\text {eff }}\right]$. For a given value of $n_{s}$, the convergence with respect to the number $\nu$ of degrees of freedom of the finite element model is given by the function $\nu \mapsto \operatorname{conv}\left(n_{s}, \nu\right)$.

\section{Application to an anisotropic random microstructure}

This section deals with the numerical application of the theory presented for a given mean model of the microstructure.

\subsection{Mean model and finite element discretization of the mean model of the microstructure}

The open bounded domain $\Omega$ (the RVE) of $\mathbb{R}^{3}$ is such that $\Omega=(] 0,1[)^{3}$. The mean model of the microstructure corresponds to a homogeneous anisotropic linear elastic medium whose local stiffness matrix $[\underline{a}]$ belonging to $M_{n}^{+}(\mathbb{R})$ with $n=6$ is then independent of $\mathbf{x}$ and such that

$$
[\underline{a}]=10^{10} \times\left[\begin{array}{cccccc}
3.3617 & 1.7027 & 1.3637 & -0.1049 & -0.2278 & 2.1013 \\
1.7027 & 1.6092 & 0.7262 & 0.0437 & -0.1197 & 0.8612 \\
1.3637 & 0.7262 & 1.4653 & -0.1174 & -0.1506 & 1.0587 \\
-0.1049 & 0.0437 & -0.1174 & 0.1319 & 0.0093 & -0.1574 \\
-0.2278 & -0.1197 & -0.1506 & 0.0093 & 0.1530 & -0.1303 \\
2.1013 & 0.8612 & 1.0587 & -0.1574 & -0.1303 & 1.7446
\end{array}\right] .
$$

The finite element model is a regular mesh of $12 \times 12 \times 12=1728$ nodes and $11 \times 11 \times 11=1331$ finite elements which are 8-nodes solid elements with $2 \times 2 \times 2$ Gauss-Legendre quadrature points (see Fig. 1). Therefore there are 5184 degrees of freedom, $N=10648$ Gauss-Legendre quadrature points, $\nu_{i}=3000$ degrees of freedom for the nodes inside domain $\Omega$ and $\nu_{b}=2184$ degrees of freedom for the nodes belonging to boundary $\partial \Omega$. In this case, the weights $\omega_{\alpha}$ introduced in Section 5.3 are such that $\omega_{\alpha}=|\Omega| / N$ and consequently, Eq. (53) can be rewritten as

$$
\mathbb{C}^{\mathrm{eff}} \simeq \frac{1}{N} \sum_{\alpha=1}^{N} \mathbb{C}\left(\mathbf{x}^{\alpha}\right): \mathbb{H}\left(\mathbf{x}^{\alpha}\right)
$$

\subsection{Meso-scale probabilistic model, computational parameters and stochastic response}

(i) Meso-scale probabilistic model. At the meso-scale, the probabilistic model of the elasticity tensor of the random anisotropic microstructure is defined in Sections 2.3, 3 and 4. The random field $\mathbf{x} \mapsto[\mathbf{A}(\mathbf{x})]$, indexed by $\Omega$, with values in $M_{n}^{+}(\mathbb{R})$, with $n=6$, is such that (see Eq. (18)), $[\mathbf{A}(\mathbf{x})]=[\underline{L}]^{T}[\mathbf{G}(\mathbf{x})][\underline{L}]$ in which the matrix $[\underline{a}]$, defined by Eq. (69), is written (see Eq. (17)) as $[\underline{a}]=[\underline{L}]^{T}[\underline{L}]$. The stochastic field $\mathbf{x} \mapsto[\mathbf{G}(\mathbf{x})]$, indexed by $\mathbb{R}^{3}$, with values in $M_{n}^{+}(\mathbb{R})$, is defined in Section 3.

(ii) Dispersion parameter. The dispersion parameter $\delta_{A}$ defined by Eq. (35) is then independent of $\mathbf{x}$ and can be calculated as a function of the parameter $\delta$ by using Eq. (36) and yields $\delta_{A}=$ $0.6192 \times \delta$. For $\delta=0.1,0.2,0.3$ and 0.4 , one then has $\delta_{A}=0.0619,0.1238,0.1858$ and 0.2477 respectively. Below, all the results are given as function of $\delta$ instead of $\delta_{A}$.

(iii) Parameters of the stochastic germs and spatial correlation lengths of the random field $[\mathbf{A}]$. In this numerical application, it is assumed that the parameters $L_{1}^{j j^{\prime}}, L_{2}^{j j^{\prime}}, L_{3}^{j j^{\prime}}$ for $1 \leq j \leq j^{\prime} \leq 6$ of the stochastic germs (see Section 3.1) are such that $L_{1}^{j j^{\prime}}=L_{2}^{j j^{\prime}}=L_{3}^{j j^{\prime}}=L_{d}$ for all $j$ and $j^{\prime}$ in which $L_{d}$ is the unique parameter relative to the length-scales of the stochastic germs. For each given value of $\delta$ and for each given value of $L_{d}$, the independent realizations of the 
random matrices $\left[\mathbf{A}\left(\mathbf{x}^{1}\right)\right], \ldots,\left[\mathbf{A}\left(\mathbf{x}^{N}\right)\right]$ at the $N=10648$ points $\mathbf{x}^{1}, \ldots, \mathbf{x}^{N}$ (see Section 5.3) are constructed by using Sections 3.3(iii) and 5.5(i). Since $L_{1}^{j j^{\prime}}=L_{2}^{j j^{\prime}}=L_{3}^{j j^{\prime}}=L_{d}$ for all $j$ and $j^{\prime}$, it can easily be deduced that the correlation function defined by Eq. (37) is such that $r^{A}(\eta, 0,0)=r^{A}(0, \eta, 0)=r^{A}(0,0, \eta)$. The following notation $r_{d}^{A}(\eta)=r^{A}(\eta, 0,0)=$ $r^{A}(0, \eta, 0)=r^{A}(0,0, \eta)$ is then used below. It can be proven that the function $r_{d}^{A}$ is independent of $\delta$. From Eq. (38), it can then be seen that the spatial correlation lengths $L_{1}^{A}, L_{2}^{A}$ and $L_{3}^{A}$ of the random field $\mathbf{x} \mapsto[\mathbf{A}(\mathbf{x})]$ are then equal to a same value denoted by $L_{A}$.

For $L_{d}=0.1$ and $L_{d}=1$, Fig. 2 and Fig. 3 display the graphs of the function $\eta \mapsto r_{d}^{A}(\eta)$ calculated with Eq. (37) in which the mathematical expectation is estimated by using the Monte Carlo simulation with 2000 independent realizations. For these values, the spatial correlation length $L_{A}$ is calculated by Eq. (38) and is such that $L_{A}=0.1113$ for $L_{d}=0.1$ and $L_{A}=1.113$ for $L_{d}=1$. More generally, one has $L_{A}=1.113 L_{d}$. Due to this correspondence between $L_{A}$ and $L_{d}$, it is equivalent to present the results in terms of the spatial correlation length $L_{A}$ or the parameter $L_{d}$. Below, the results are presented in function of $L_{d}$.

Let $\mathcal{C}_{d}$ be the cube $L_{A} \times L_{A} \times L_{A}$. For the smallest value of $L_{A}$ considered in the numerical calculation $\left(L_{A}=0.1113\right.$ corresponding to $\left.L_{d}=0.1\right)$ there are about 14 Gauss-Legendre quadrature points in $\mathcal{C}_{d}$. For $L_{A}=0.2226$ corresponding to $L_{d}=0.2$, there are about 112 points. This is sufficient to obtain a good approximation of the random matrix $[\mathbf{K}]$ by $\left[\mathbf{K}_{N}\right]$ in the mean-square sense (see Section 5.3-(ii)) taking into account the very slow variations (over any interval of length $L_{A}$ ) of the correlation function displays in Fig. 2. In addition, Fig. 5 shows that the convergence is reached with respect to $N$ for this smallest value of $L_{A}$ and for the strongest stochastic fluctuations considered $(\delta=0.4)$. This is an additional important information to conclude that the convergence is reached in the mean-square sense with a resonable accuracy.

(iv) Stochastic convergence analysis for the random effective stiffness matrix. For each given value of the dispersion parameter $\delta$, for each given value of the parameter $L_{d}$ and for a given finite element model having $\nu$ degrees of freedom, the probabilistic quantities (defined in Section 5.4) for the random effective stiffness matrix are estimated by using the Monte Carlo numerical simulation presented in Section 5.5 and by using the equations given in Sections 5.3 and 5.4.

(iv-1) The mean-square convergence with respect to $n_{s}$ (number of realizations used in the Monte Carlo numerical method) is then studied by constructing the function $n_{s} \mapsto \operatorname{conv}\left(n_{s}, \nu\right)$ defined by Eq. (68). Fig. 4 displays the graph of $n_{s} \mapsto \operatorname{conv}\left(n_{s}, \nu\right)$ for $\delta=0.4$ (the largest value considered for the dispersion parameter), for $L_{d}=0.1$ (the smallest value considered for this parameter) and for a finite element model having $\nu=5184$ degrees of freedom and corresponding to $11 \times 11 \times 11$ finite elements. It can be seen that convergence is reached for $n_{s} \geq 500$.

(iv-2) The mean-square convergence with respect to the number $\nu$ of degrees of freedom of the finite element model is studied by constructing the function $\nu \mapsto \operatorname{conv}\left(n_{s}, \nu\right)$ defined by Eq. (68). Fig. 5 displays the graph of $\nu \mapsto \operatorname{conv}\left(n_{s}, \nu\right)$ for $\delta=0.4, L_{d}=0.1$ and $n_{s}=900$. Clearly, convergence is reached for $11 \times 11 \times 11$ finite elements corresponding to $\nu=5184$.

(iv-3) All the results presented below have been computed with $n_{s}=900$ and $11 \times 11 \times 11$ finite elements corresponding to $\nu=5184$. Convergence is reached for the values of $L_{d}$ and $\delta$ which are considered below.

(v) Normalized mean value of the norm of the random effective stiffness matrix. Let $\|\underline{a}\|$ be the norm (defined by Eq. (30)) of the local stiffness matrix $[\underline{a}]$ which is such that $\|\underline{a}\|=\underline{\lambda}_{1}$ where $\underline{\lambda}_{1}$ is the largest eigenvalue of the matrix $[\underline{a}]$. The normalized mean value $\mu_{\| \text {Aff }_{\|}}$of the random 
variable $\left\|\mathbf{A}^{\text {eff }}\right\|$ (see Eq. (30)) is then defined by

$$
\mu_{\left\|\mathrm{A}_{\mathrm{eff}}\right\|}=\frac{m_{\left\|\mathrm{A}_{\mathrm{eff}}\right\|}}{\|\underline{a}\|},
$$

in which the mean value $m_{\| \text {eff }_{\|}}$is defined by Eq. (61). Fig. 6 shows $\mu_{\left\|A^{\text {eff }}\right\|}$ as a function of the parameter $L_{d}$ for several values of the dispersion parameter $\delta$. Fig. 6 displays the graph of the function $L_{d} \mapsto \mu_{\| \mathrm{A}_{\text {eff } \|}}\left(L_{d}\right)$ for $L_{d}$ belonging to the interval $[0.1,1.0]$ and for $\delta=0.1$ (circle marker), $\delta=0.2$ (square marker), $\delta=0.3$ (cross marker) and $\delta=0.4$ (diamond marker).

(vi) Coefficient of variation of the random norm of the random effective stiffness matrix. This coefficient of variation $\mathrm{cv}_{\left\|A_{\text {eff }}\right\|}$ is defined by Eq. (63). Fig. 7 shows the coefficient of variation $\mathrm{cv}_{\| \mathrm{A}_{\text {eff } \|}}\left(L_{d}\right)$ of the random norm of the random effective stiffness matrix as a function of the parameter $L_{d}$, for several values of the dispersion parameter $\delta$. Fig. 7 displays the graph of the function $L_{d} \mapsto \mathrm{cv}_{\| \mathrm{A}_{\text {eff } \|}}\left(L_{d}\right)$ for $L_{d}$ belonging to the interval $[0.1,1.0]$ and for $\delta=0.1$ (circle marker), $\delta=0.2$ (square marker), $\delta=0.3$ (cross marker) and $\delta=0.4$ (diamond marker).

(vii) Cumulative distribution function of the random variable $Z$ related to the random effective stiffness matrix. Fig. 8 shows the cumulative distribution function $F_{Z}$ (defined by Eq. (66)) of the normalized random variable $Z$ (defined by Eq. (65)) for several values of the spatial correlation length $L_{d}$ and for $\delta=0.4$. Fig. 8 displays the graph of $z^{*} \mapsto F_{Z}\left(z^{*}\right)=P\left\{Z \leq z^{*}\right\}$ for $L_{d}$ belonging to the interval $[0.1,1.0]$ and for $\delta=0.4$. Fig. 9 displays the graph of $z^{*} \mapsto 1-F_{Z}\left(z^{*}\right)=P\left\{Z>z^{*}\right\}$ for the same values of $L_{d}$ and $\delta$.

(viii) Mean value and coefficient of variation of the random effective stiffness matrix. For $\delta=0.4$ and for $L_{d}=0.1$, the mean value $\left[m_{\mathbf{A}^{\text {eff }}}\right]$ defined by Eq. (58) and the coefficient of variation $\left[\mathrm{cv}_{\mathbf{A}^{\text {eff }}}\right]$ defined by Eq. (60) of the components $\left[\mathbf{A}^{\text {eff }}\right]_{I J}$ of the random effective stiffness matrix $\left[\mathbf{A}^{\text {eff }}\right]$ are such that

$$
\begin{gathered}
{\left[m_{\mathbf{A}^{\text {eff }}}\right]=10^{10} \times\left[\begin{array}{cccccc}
3.1917 & 1.6169 & 1.2936 & -0.0991 & -0.2165 & 1.9921 \\
1.6169 & 1.5348 & 0.6899 & 0.0422 & -0.1138 & 0.8159 \\
1.2936 & 0.6899 & 1.3991 & -0.1120 & -0.1437 & 1.0053 \\
-0.0991 & 0.0422 & -0.1120 & 0.1266 & 0.0089 & -0.1497 \\
-0.2165 & -0.1138 & -0.1437 & 0.0089 & 0.1468 & -0.1236 \\
1.9921 & 0.8159 & 1.0053 & -0.1497 & -0.1236 & 1.6566
\end{array}\right] .} \\
{\left[\operatorname{cv}_{\mathbf{A}^{\text {eff }}}\right]=\left[\begin{array}{cccccc}
0.0174 & 0.0204 & 0.0237 & 0.0742 & 0.0403 & 0.0186 \\
0.0204 & 0.0166 & 0.0288 & 0.1267 & 0.0505 & 0.0264 \\
0.0237 & 0.0288 & 0.0166 & 0.0440 & 0.0394 & 0.0223 \\
0.0742 & 0.1267 & 0.0440 & 0.0165 & 0.1705 & 0.0361 \\
0.0403 & 0.0505 & 0.0394 & 0.1705 & 0.0170 & 0.0494 \\
0.0186 & 0.0264 & 0.0223 & 0.0361 & 0.0494 & 0.0170
\end{array}\right] .}
\end{gathered}
$$

For $\delta=0.4$ and for $L_{d}=1$, one has

$$
\left[m_{\mathbf{A}^{\text {eff }}}\right]=10^{10} \times\left[\begin{array}{cccccc}
3.3187 & 1.6872 & 1.3438 & -0.1065 & -0.2265 & 2.0767 \\
1.6872 & 1.6055 & 0.7220 & 0.0448 & -0.1171 & 0.8488 \\
1.3438 & 0.7220 & 1.4576 & -0.1161 & -0.1502 & 1.0413 \\
-0.1065 & 0.0448 & -0.1161 & 0.1314 & 0.0096 & -0.1578 \\
-0.2265 & -0.1171 & -0.1502 & 0.0096 & 0.1503 & -0.1298 \\
2.0767 & 0.8488 & 1.0413 & -0.1578 & -0.1298 & 1.7255
\end{array}\right]
$$




$$
\left[\mathrm{cv}_{\mathbf{A}^{\mathrm{eff}}}\right]=\left[\begin{array}{llllll}
0.1793 & 0.2153 & 0.2385 & 0.7723 & 0.4223 & 0.1941 \\
0.2153 & 0.1765 & 0.2946 & 1.2696 & 0.5328 & 0.2762 \\
0.2385 & 0.2946 & 0.1820 & 0.4935 & 0.4202 & 0.2294 \\
0.7723 & 1.2696 & 0.4935 & 0.1715 & 1.8137 & 0.4041 \\
0.4223 & 0.5328 & 0.4202 & 1.8137 & 0.1828 & 0.5171 \\
0.1941 & 0.2762 & 0.2294 & 0.4041 & 0.5171 & 0.1818
\end{array}\right]
$$

\subsection{Probabilistic analysis of the RVE size}

The probability analysis of the RVE size can be deduced from Figs. 7, 8 and 9. Stochastic homogenization can be performed if the random fluctuations of $\left\|\mathbf{A}^{\text {eff }}\right\|$ around its mean value $m_{\left\|\mathbf{A}^{\text {eff }}\right\|}$ is very small, that is to say if $\left\|\mathbf{A}^{\text {eff }}\right\|$ has values around 1.

(i) In terms of the coefficient of variation $\mathrm{cv}_{\left\|\mathbf{A}^{\text {eff }}\right\|}$ of the random variable $\left\|\mathbf{A}^{\text {eff }}\right\|$, the REV size has to be small with respect to the parameter $L_{d}$, i.e $\mathrm{cv}_{\| \text {eff } \|} \ll 1$. For instance, Fig. 7 shows that if $L_{d} \leq 0.72$ for $\delta=0.1, L_{d} \leq 0.35$ for $\delta=0.2, L_{d} \leq 0.24$ for $\delta=0.3$ or $L_{d} \leq 0.18$ for $\delta=0.4$, then $\mathrm{cv}_{\| \mathrm{A} \text { eff }} \leq 0.04$. For instance, for $\delta=0.4$ (i.e. $\delta_{A}=0.2477$ ), this means that if stochastic homogenization is performed with a RVE whose size is about five times the spatial correlation length (REV size $=1$ and parameter $L_{d}=0.18$ ), then the normalized standard deviation of the random fluctuations of the effective stiffness tensor is less than $4 \%$. Nevertheless, Eq. (74) shows that the normalized standard deviation of the components $\left[\mathbf{A}^{\text {eff }}\right]_{I J}$ can be much more larger than $4 \%$. For instance, one has $18 \%$ for $(1,1)$ and $77 \%$ for $(1,4)$.

(ii) The above second-order analysis can be improved in studying the probability distributions. Let $\beta$ be a positive real number. Let $\beta \mapsto \mathbb{P}(\beta)$ be the function defined by

$$
\mathbb{P}(\beta)=P\{1-\beta<Z \leq 1+\beta\}=F_{Z}(1+\beta)-F_{Z}(1-\beta)
$$

In terms of probability, this information is given by the function $\mathbb{P}$ defined by Eq. (75) and is deduced from $F_{Z}$. For $\delta=0.4$ and for $L_{d}$ belonging to [0.1, 0.7], Fig. 10 displays the graph of the function $\mathbb{P}$. For instance, for $L_{d}=0.2$ and for $\beta=0.02,0.04$ and 0.08 , one has $\mathbb{P}=0.36,0.65$ and 0.95 , which means that $P\{0.98<Z \leq 1.02\}=0.36, P\{0.96<Z \leq 1.04\}=0.65$ and $P\{0.92<Z \leq 1.08\}=0.95$. For instance, for $\delta=0.4$ (i.e. $\delta_{A}=0.2477$ ), if stochastic homogenization is performed with a RVE whose size is five times the spatial correlation length (REV size $=1$ and spatial correlation length $L_{A}=0.2226$ ), then the probability for which the random fluctuations of the effective stiffness tensor is less than $2 \%, 4 \%$ or $8 \%$, is $0.36,0.65$ or 0.95 respectively.

\section{Summary of the proposed theory}

The proposed theory is devoted to stochastic homogenization of a random anisotropic elastic microstructure which cannot be described in terms of its constituents and then for which the standard method cannot be applied (for instance some cortical bones, some biological membranes and more generally, some living tissues, etc). The methodology consists in directly introducing a meso-scale probabilistic model of the random anisotropic elastic microstructure (which is not deduced from the probabilistic models of its constituents). For this meso-scale model, the local stiffness fourth-order tensor-valued random field is $\left\{\mathbb{C}_{i j k h}(\mathbf{x})\right\}_{i j k h}$ and is represented by a $(6 \times 6)$ real random matrix $[\mathbf{A}(\mathbf{x})]$. Its mean value $\left\{\underline{\mathbb{C}}_{i j k h}(\mathbf{x})\right\}_{i j k h}$ is represented by a $(6 \times 6)$ real matrix $[\underline{a}(\mathbf{x})]$. This meso-scale model allows a complete stochastic model of the fourth-order random effective (macroscopic) stiffness tensor $\mathbb{C}^{\text {eff }}$ related to a given Representative Volume 
Element (RVE) to be constructed. This random effective stiffness tensor $\mathbb{C}^{\text {eff }}$ is represented by a $(6 \times 6)$ real random matrix $\left[\mathbf{A}^{\text {eff }}\right]$. Three types of analysis can be performed with the theory proposed.

(1) The first type of analysis corresponds to a direct prediction of $\left[\mathbf{A}^{\text {eff }}\right]$. For such a prediction, data are (a) the size of the RVE; (b) the mean value $[\underline{a}(\mathbf{x})]$ ) of the local stiffness fourth-order tensor-valued random field associated with the mean model of the meso-scale probabilistic model of the random anisotropic elastic microstructure; (c) the parameters describing the $(6 \times 6)$ real random matrix $[\mathbf{A}(\mathbf{x})]$ which are the dispersion parameter $\delta_{A}$ and the correlation lengths $L_{1}^{A}, L_{2}^{A}, L_{3}^{A}$. The methodology then consists (a) in constructing $n_{s}$ independent realizations $\left[\mathbf{A}\left(\mathbf{x}, \theta_{1}\right)\right], \ldots,\left[\mathbf{A}\left(\mathbf{x}, \theta_{n_{s}}\right)\right]$ for $\theta_{1}, \ldots, \theta_{n_{s}}$ in $\Theta$, using Eqs. (17) and (18) and Section 3.3; (b) in solving random equations (52) to (57) by the Monte Carlo numerical simulation method by using the $n_{s}$ independent realizations $\left[\mathbf{A}\left(\mathbf{x}, \theta_{1}\right)\right], \ldots,\left[\mathbf{A}\left(\mathbf{x}, \theta_{n_{s}}\right)\right] ;$ (c) in constructing the probabilistic quantities (for instance, the moments and the probability distribution) of the random matrix $\left[\mathbf{A}^{\text {eff }}\right]$ representing the fourth-order random effective stiffness, using Eqs. (58) to (66).

(2) The second type is a probability analysis of the RVE size with respect to the spatial correlation lengths of the proposed meso-scale probabilistic model. The parametric study of the RVE size can be performed in function of the dispersion parameter $\delta_{A}$ and the correlation lengths $L_{1}^{A}, L_{2}^{A}, L_{3}^{A}$ of the random field $\mathbf{x} \mapsto[\mathbf{A}(\mathbf{x})]$. Such a study is also useful to get information on the RVE size for which stochastic fluctuations are still significant and consequently, can be measured. Such an analysis is carried out using the direct prediction of the probabilistic quantities of the fourth-order random effective stiffness tensor as explained in point (1) above.

(3) The third type of analysis is related to the experimental identification of the meso-scale probabilistic model of the random anisotropic elastic microstructure carried out in identifying the parameters of the random field $\mathbf{x} \mapsto[\mathbf{A}(\mathbf{x})]$. Such an experimental identification can be performed using field measurements deduced from digital image processing and solving an inverse stochastic problem.

\section{Conclusions}

We have proposed a new approach useful for a direct experimental identification of random anisotropic elastic microstructures introducing a meso-scale probabilistic model. Such an approach can be used when the standard method cannot easily be applied to anisotropic elastic microstructures. A parametric probabilistic study of the RVE size has been performed with respect to the intensity $\delta_{A}$ of the stochastic fluctuations of the local stiffness tensor-valued random field describing the meso-scale probabilistic model and in function of its correlation lengths $L_{k}^{A}$. Such a study is also useful to get information on the RVE size for which stochastic fluctuations are still significant and consequently, can be measured. Then the results presented in this paper could allow the meso-scale probabilistic model to be identified from meso- or macro-scale measurements solving an inverse stochastic problem. For fixed spatial correlation lengths, the RVE size has to increase with $\delta_{A}$. For a fixed value of $\delta_{A}$, the RVE size has to increase with the spatial correlation lengths. For fixed spatial correlation lengths and dispersion parameter $\delta_{A}$, the effective RVE size can effectively be calculated in a probability sense using the cumulative distribution function of the random variable measuring the random fluctuations of the effective stiffness tensor. A large numerical simulation has been carried out and the numerical results obtained allow a probability analysis of the representative volume element size to be performed. 


\section{References}

Andraud, C., Geghdadi, A., Haslund, E., Hilfer, R., Lafait, J., Virgin, B., 1997. Local entropy characterization of correlated random microstructures. Physica A 235, 307-318.

Andrews, K.T., Wright, S., 1998. Stochastic homogenization of elliptic boundray-value problems with $\ell^{p}$ data. Asymptotic Analysis 17, 165-184.

Beran, M.J., McCoy, J.J., 1970. Mean field variations in a statistical sample of heterogeneous linearly elastic solids. Int. J. Solids Structures 6, 1035-1054.

Beran, M.J., McCoy, J.J., 1970. The use of strain gradient theory for analysis of random media. Int. J. Solids Structures 6, 1267-1275.

Bourgeat, A., Piatnitski, A., 2004. Approximations of effective coefficients in stochastic homogenization. Annales de l'Institut Henri Poincaré, Probabilité et Statistiques PR 40, 153-165.

Drugan, W.J., Willis, J.R., 1996. A micromechanics-based nonlocal constitutive equation and estimates of representative volume element size for elastic composites. Journal of the Mechanics and Physics of Solids 44(4), 497-524.

Jikov, V.V., Kozlov, S.M., Oleinik, O.A., 1994. Homogenization of differential operators and integral functionals. Springer-Verlag, New-York.

Kachanov, M., Sevostianov, I., Shafiro, B., 2001. Explicit cross-property correlations for porous materials with anisotropic microstructures. Journal of the Mechanics and Physics of Solids 49, 1-25.

Krée, P., Soize, C. 1986. Mathematics of random phenomena. Reidel, Dordrecht.

Kröner, E., 1971. Statistical continuum mechanics. Springer-Verlag, Wien.

Kröner, E., 1977. Bounds for effective elastic moduli of disordered materials. Journal of the Mechanics and Physics of Solids 25, 137-155.

Milton, G.W., 2002. The theory of composites. Cambridge University Press, Cambridge.

Nemat-Nasser, S., Hori, M., 1999. Micromechanics: overall properties of heterogeneous materials. Second revised edition. Elsevier, Amsterdam.

Ostoja-Starzewski, M., 1998. Random field models of heterogeneous materials. International Journal of Solids and Structures 35, 2429-2455.

Papanicolaou, G., Varadhan, S.R.S., 1981. Boundary value problems with rapidly oscillating random coefficients. In: Fritz, J., Lebowitz, J.L., Szász, D. (Eds.), Proceedings of Colloquium on Random fields, Rigorous results in statistical mechanics and quantum field theory, Colloquia Mathematica Societatis Janos Bolyai 27, North-Holland, Amsterdam, pp. 835-873.

Poirion, F., Soize, C., 1995. Numerical methods and mathematical aspects for simulation of homogeneous and non homogeneous Gaussian vector fields, pp. 17-53 in Probabilistic Methods in Applied Physics, Krée, P. and Wedig, W. (Eds.), Lecture Notes in Physics, ISBN 3-54060214-3, Springer-Verlag, Berlin.

Quintanilla, J., 1999. Microstructure functions for random media with impenetrable particles. Physical Review E 60, 5788-5794.

Ren, Z.-Y., Zheng, Q.-S., 2004. Effects of grain sizes, shapes, and distribution on minimum sizes of representative volume elements of cubic polycrystals. Mechanics of Materials 36, 1217-1229.

Sab, K., 1992. On the homogenization and the simulation of random materials. European Journal of Mechanics, A/Solids 11(5), 585-607. 
Sab, K., Nedjar, B., 2005. Periodization of random media and representative volume element size for linear composites. Comptes Rendus Mécanique 333, 187-195.

Sanchez-Palencia, E., 1980. Non-homogeneous media and vibration theory. Lecture Notes in Physics 127. Springer-Verlag, Berlin.

Serfling, R.J., 1980. Approximation theorems of mathematical statistics. John Wiley \& Sons.

Shinozuka, M., 1971. Simulations of multivariate and multidimensional random processes. Journal of Acoustical Society of America 39(1), 357-367.

Soize, C., 2001. Maximum entropy approach for modeling random uncertainties in transient elastodynamics. Journal of Acoustical Society of America 109(5), 1979-1996.

Soize, C., 2004. Random-field model for the elasticity tensor of anisotropic random media. Comptes Rendus Mécanique 332, 1007-1012.

Soize, C., 2006. Non Gaussian positive-definite matrix-valued random fields for elliptic stochastic partial differential operators. Computer Methods in Applied Mechanics and Engineering 195(1-3), 26-64.

Torquato,S., Stell, G., 1985. Microstructure of two-phase random media. V. The $n$-point matrix probability functions for impenetrable spheres. J. Chem. Phys. 82, 980-987.

Torquato, S., 1997. Effective stiffness tensor of composite media - I. Exact series expansions. Journal of the Mechanics and Physics of Solids 45(9), 1421-1448.

Torquato, S., 2002. Random heterogeneous materials, microstructure and macroscopic properties. Springer-Verlag, New York.

Zaoui, A., 2002. Continuum micromechanics: Survey. Journal of Engineering Mechanics 128 (8), 808-816. 


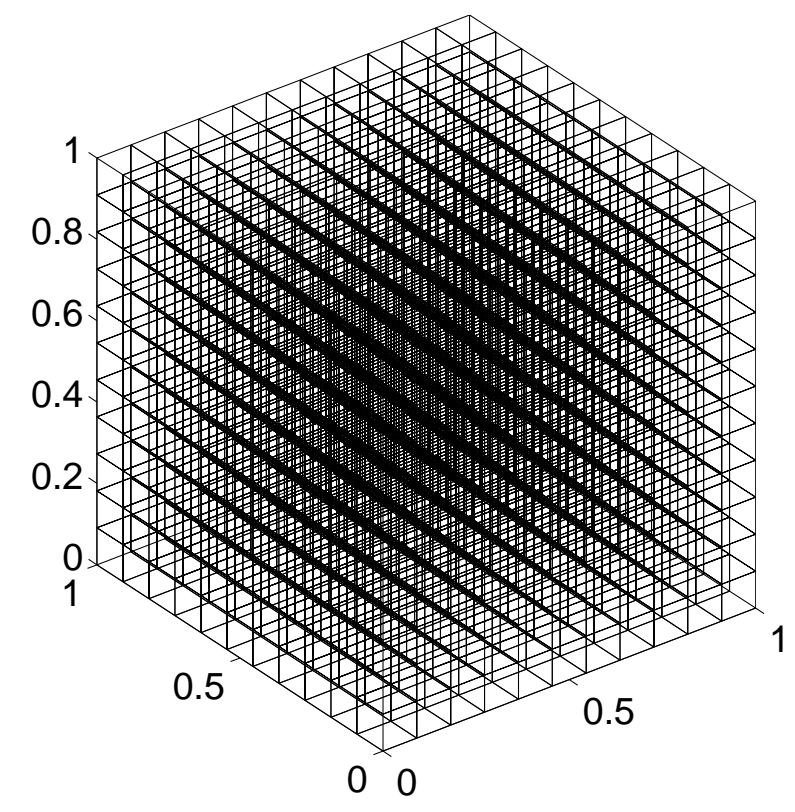

Fig. 1. Finite element model of the Representative Volume Element

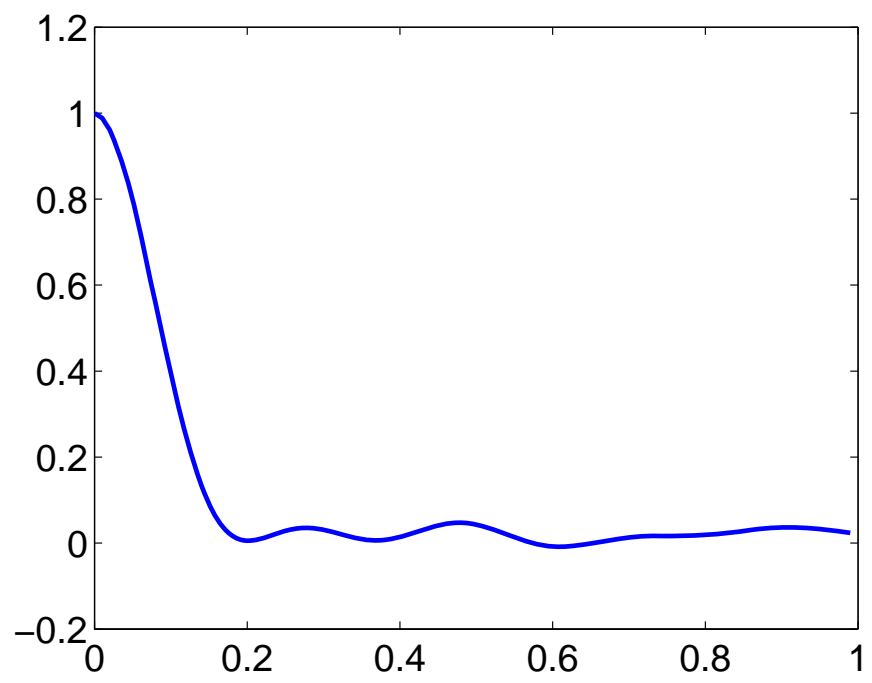

Fig. 2. Graph of the correlation function $\eta \mapsto r_{d}^{A}(\eta)$ for $L_{d}=0.1$. Horizontal axis $\eta$. Vertical axis $r_{d}^{A}(\eta)$ 


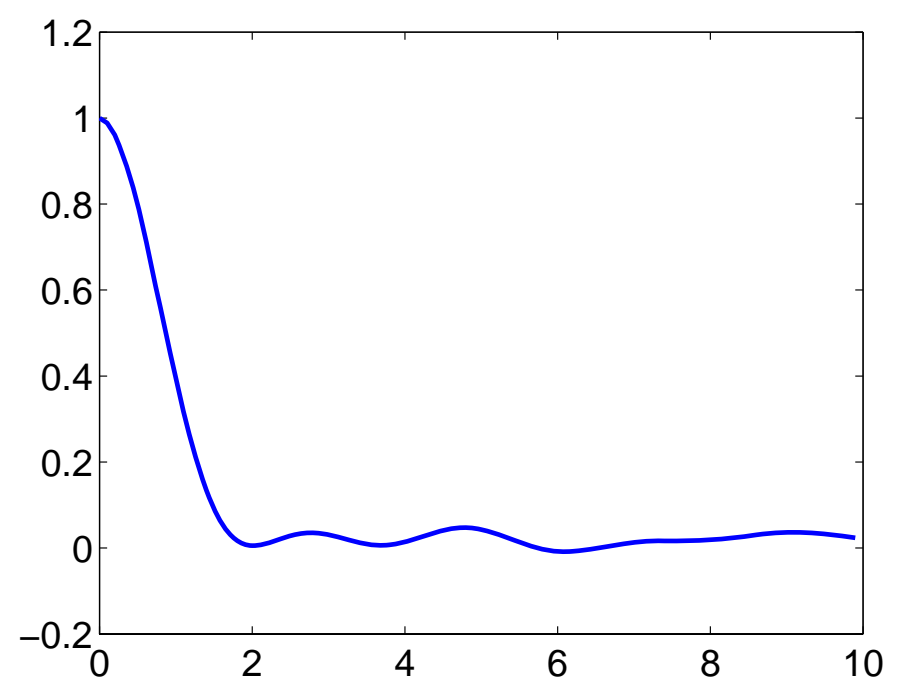

Fig. 3. Graph of the correlation function $\eta \mapsto r_{d}^{A}(\eta)$ for $L_{d}=1.0$. Horizontal axis $\eta$. Vertical axis $r_{d}^{A}(\eta)$

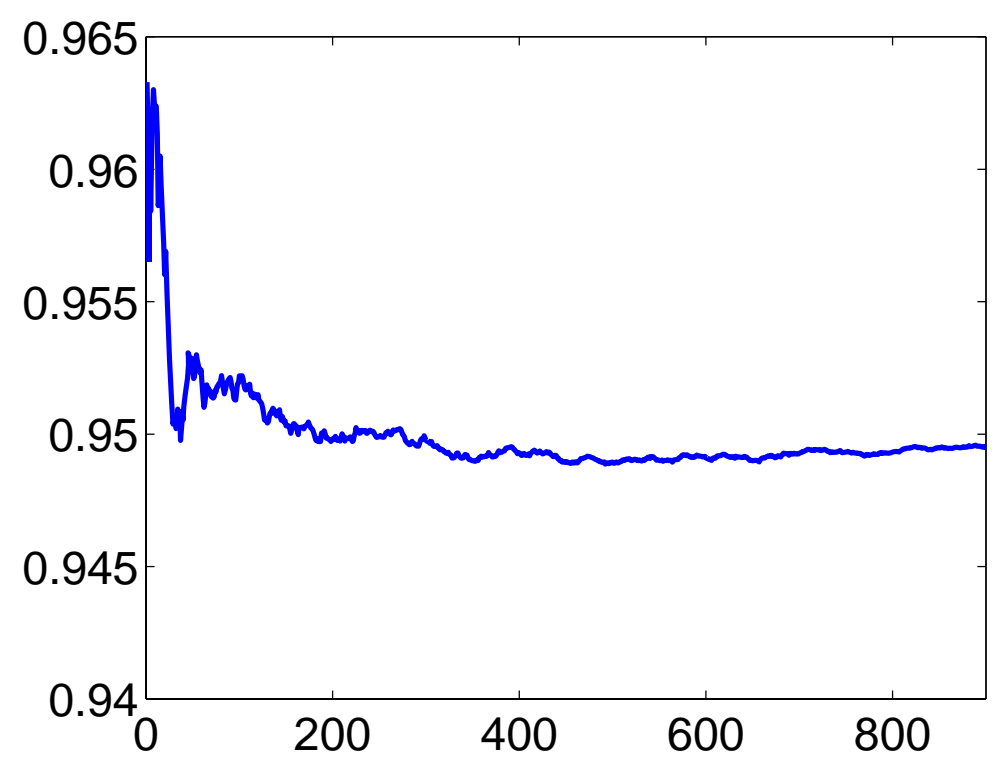

Fig. 4. Mean-square convergence of the random effective stiffness matrix with respect to the number $n_{s}$ of realizations of the Monte Carlo numerical simulation method for $\delta=0.4$, $L_{d}=0.1$ and $\nu=5184$. Graph of function $n_{s} \mapsto \operatorname{conv}\left(n_{s}, \nu\right)$. Horizontal axis $n_{s}$. Vertical axis $\operatorname{conv}\left(n_{s}, \nu\right)$. 


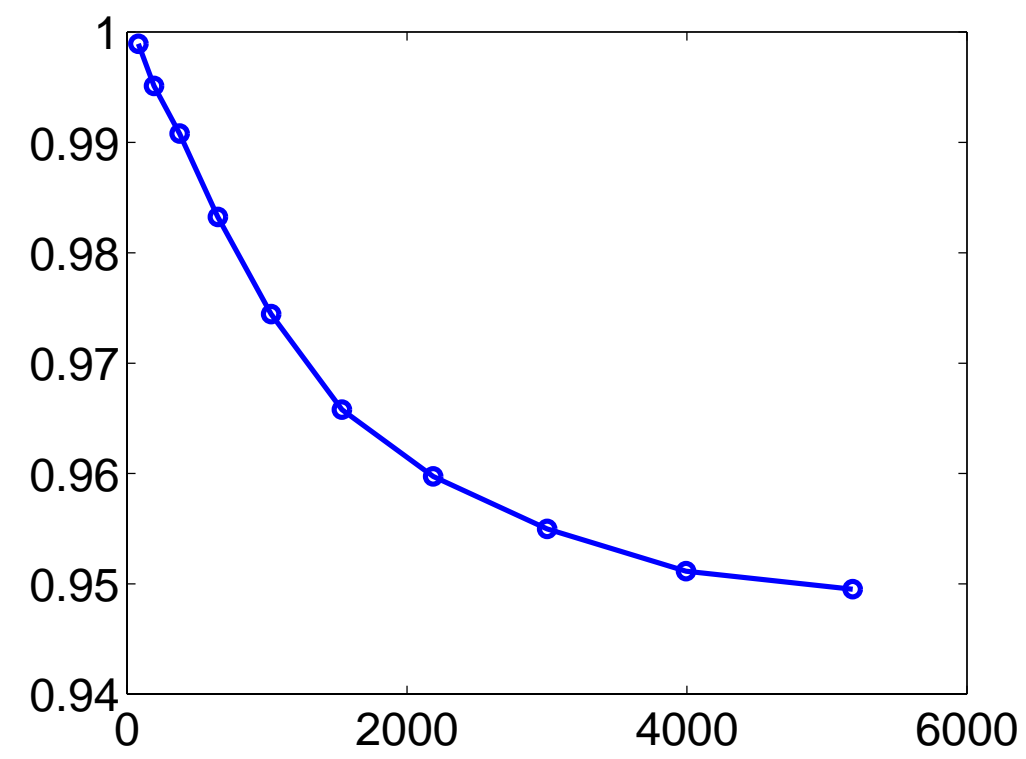

Fig. 5. Mean-square convergence of the random effective stiffness matrix with respect to the number $\nu$ of degrees of freedom of the finite element model for $\delta=0.4, L_{d}=0.1$ and for $n_{s}=900$. Graph of function $\nu \mapsto \operatorname{conv}\left(n_{s}, \nu\right)$. Horizontal axis $\nu$. Vertical axis $\operatorname{conv}\left(n_{s}, \nu\right)$.

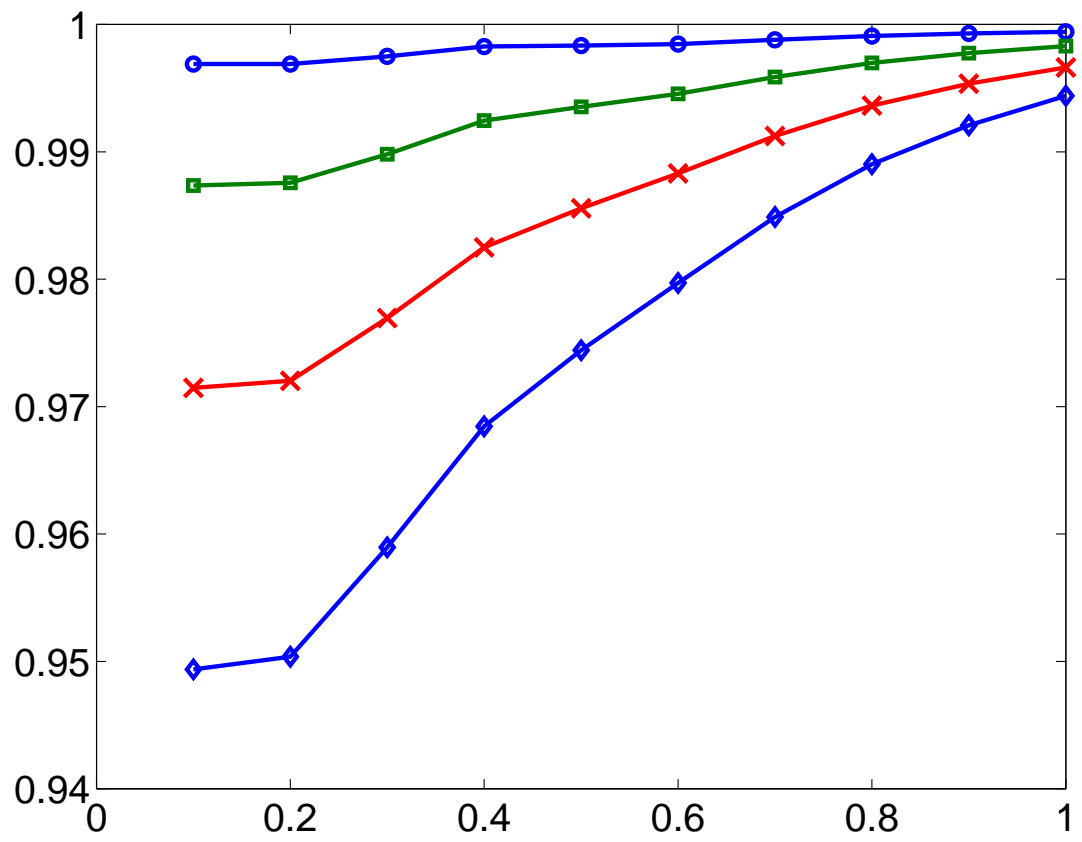

Fig. 6. Normalized mean value $\mu_{\|\mathbb{A}\|}\left(L_{d}\right)$ (vertical axis) of the random norm $\|\mathbb{A}\|$ of the random effective stiffness matrix $[\mathbb{A}]$ as a function of the spatial correlation length $L_{d}$ (horizontal axis). Graph of function $L_{d} \mapsto \mu_{\|\mathbb{A}\|}\left(L_{d}\right)$ for $\delta=0.1$ (circle marker), $\delta=0.2$ (square marker), $\delta=0.3$ (cross marker) and $\delta=0.4$ (diamond marker). 


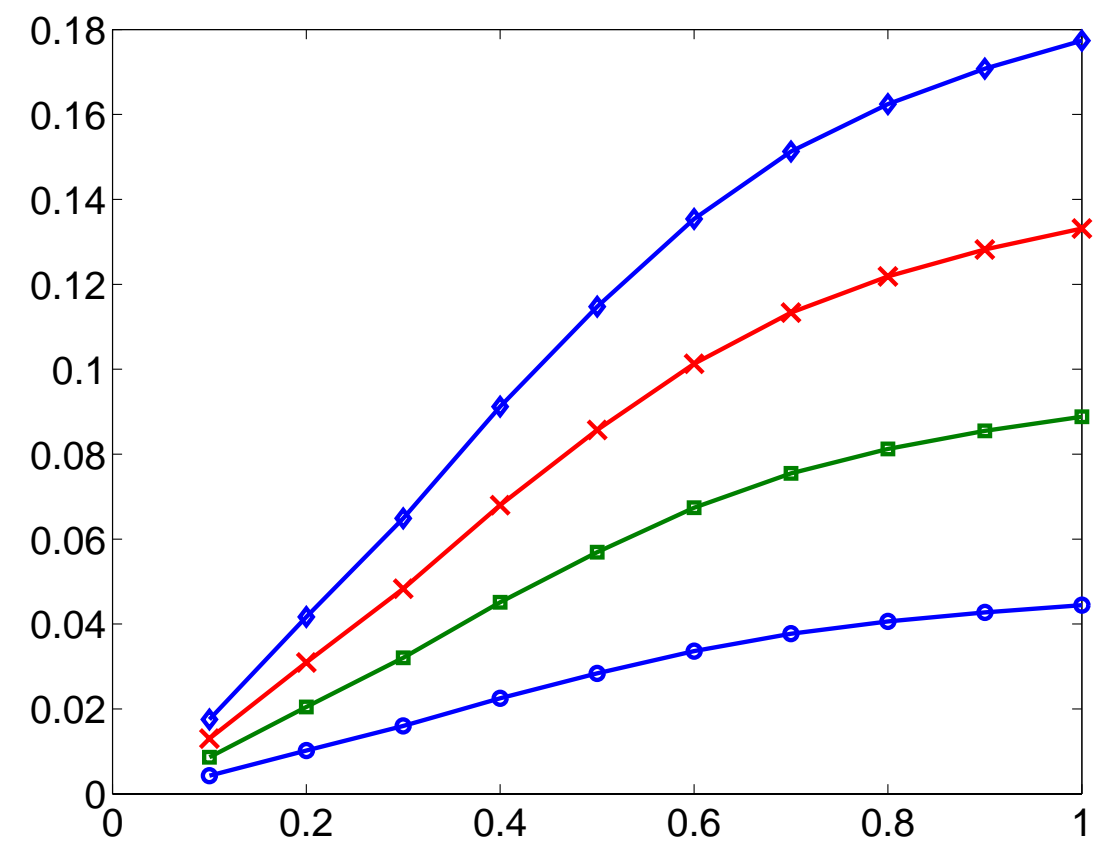

Fig. 7. Coefficient of variation $\mathrm{cv}_{\|\mathbb{A}\|}\left(L_{d}\right)$ (vertical axis) of the random norm $\|\mathbb{A}\|$ of the random effective stiffness matrix $[\mathbb{A}]$ as a function of the spatial correlation length $L_{d}$ (horizontal axis). Graph of function $L_{d} \mapsto \mathrm{cv}_{\|\mathbb{A}\|}\left(L_{d}\right)$ for $\delta=0.1$ (circle marker), $\delta=0.2$ (square marker), $\delta=0.3$ (cross marker) and $\delta=0.4$ (diamond marker).

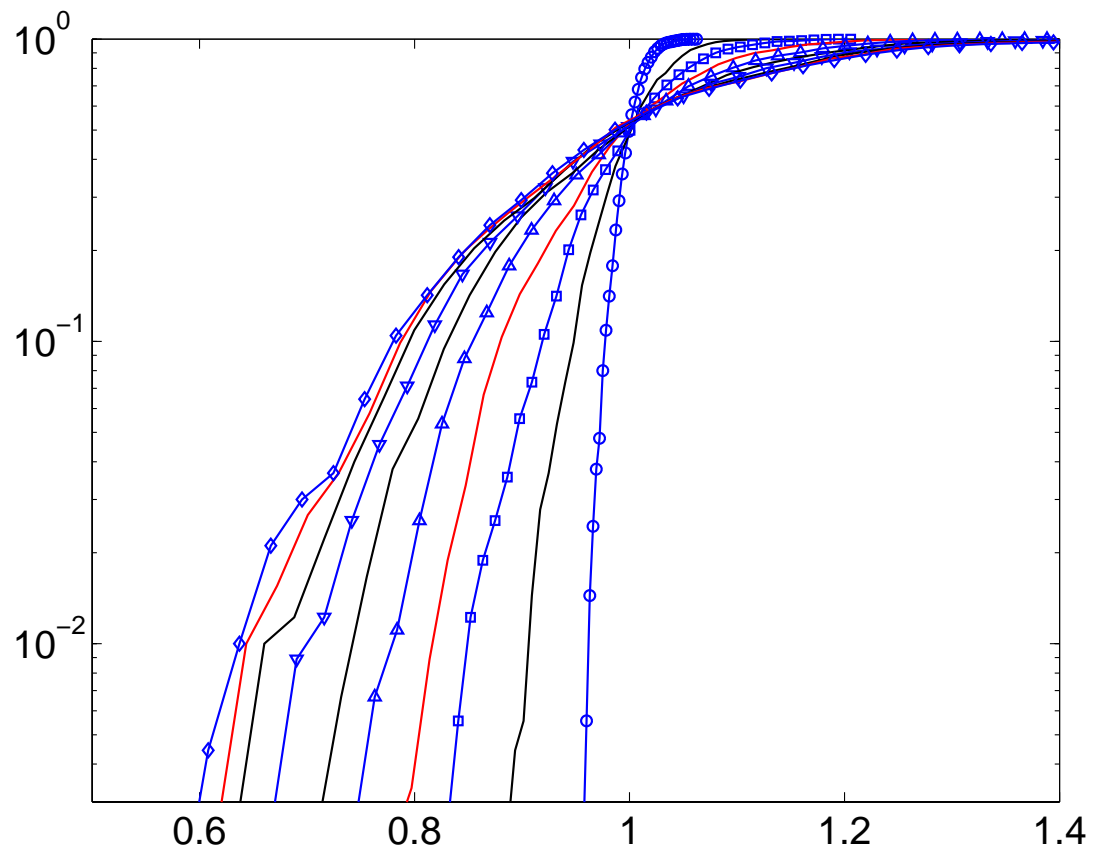

Fig. 8. Graph of the cumulative distribution function $z^{*} \mapsto F_{Z}\left(z^{*}\right)=P\left\{Z \leq z^{*}\right\}$ of the normalized random variable $Z=\|\mathbb{A}\| / m_{\|\mathbb{A}\|}$ for $\delta=0.4$ and for several values of the spatial correlation length $L_{d}=0.1$ (circle), 0.2 (no marker), 0.3 (square), 0.4 (no marker), 0.5 (triangleup), 0.6 (no marker), 0.7 (triangle-down), 0.8 (no marker), 0.9 (no marker), 1.0 (diamond). Horizontal axis $z^{*}$. Vertical axis $F_{Z}\left(z^{*}\right)$ in $\log _{10}$ scale. 


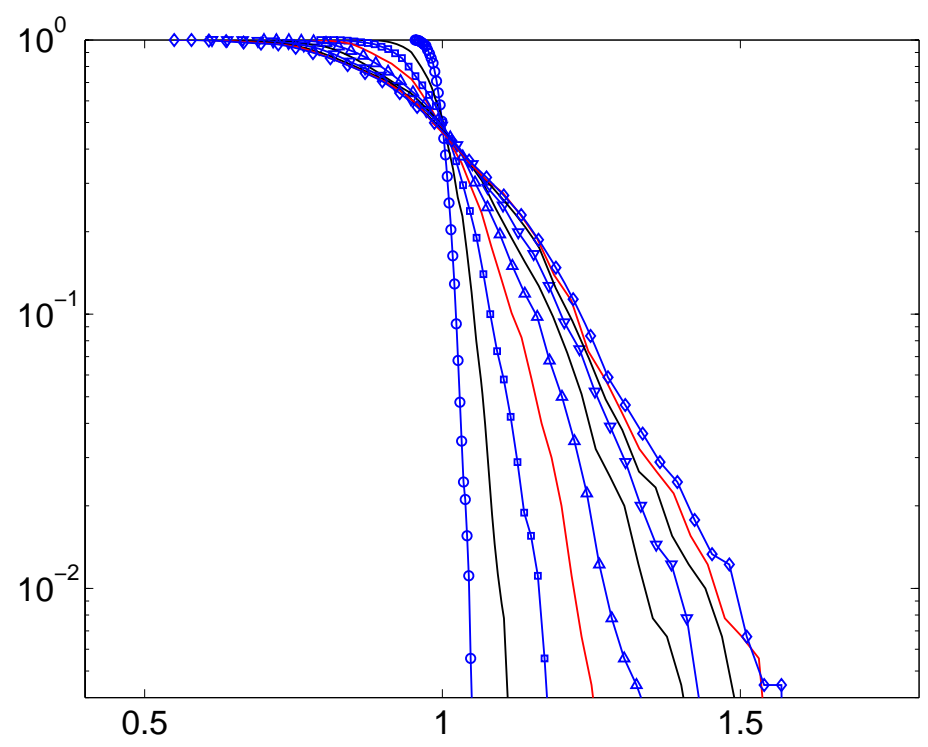

Fig. 9. Graph of $z^{*} \mapsto 1-F_{Z}\left(z^{*}\right)=P\left\{Z>z^{*}\right\}$ relative to the normalized random variable $Z=\|\mathbb{A}\| / m_{\|\mathbb{A}\|}$ for $\delta=0.4$ and for several values of the spatial correlation length $L_{d}=0.1$ (circle), 0.2 (no marker), 0.3 (square), 0.4 (no marker), 0.5 (triangle-up), 0.6 (no marker), 0.7 (triangle-down), 0.8 (no marker), 0.9 (no marker), 1.0 (diamond). Horizontal axis $z^{*}$. Vertical axis $F_{Z}\left(z^{*}\right)$ in $\log _{10}$ scale.

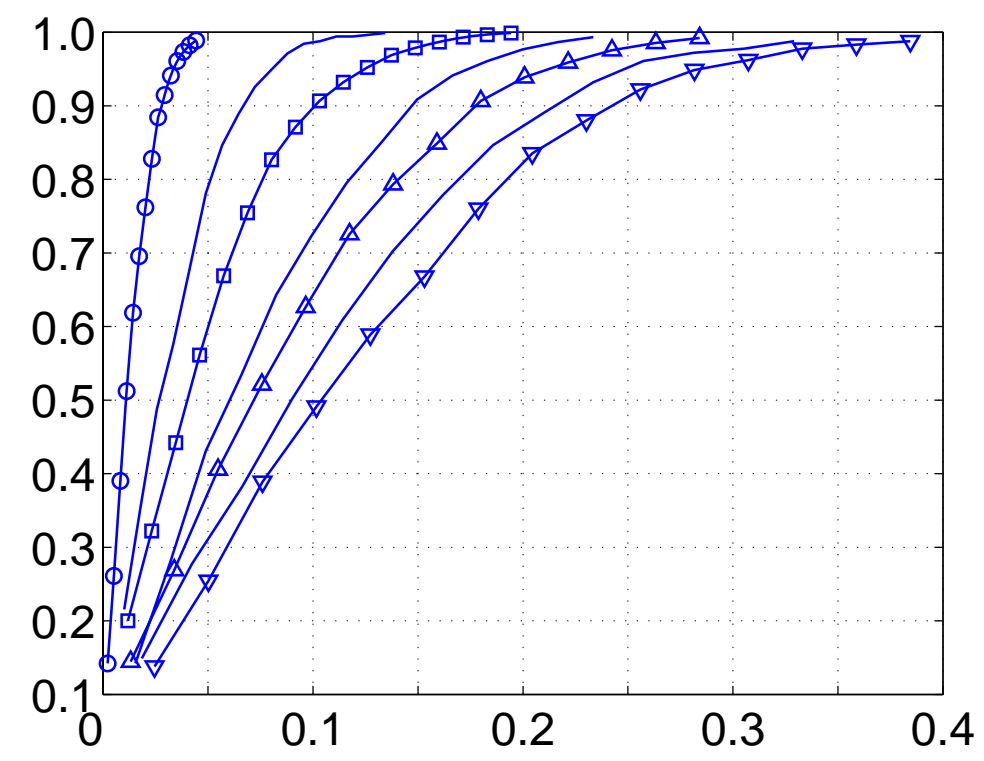

Fig. 10. Graph of $\beta \mapsto \mathbb{P}(\beta)=P\{1-\beta<Z \leq 1+\beta\}$ for $\delta=0.4$ and for several values of the spatial correlation length $L_{d}=0.1$ (circle), 0.2 (no marker), 0.3 (square), 0.4 (no marker), 0.5 (triangle-up), 0.6 (no marker), 0.7 (triangle-down). Horizontal axis $\beta$. Vertical axis $\mathbb{P}(\beta)$. 